This is the final peer-reviewed accepted manuscript of:

Franca, M. (2020). Corrigendum and addendum to "non-autonomous quasilinear elliptic equations and Ważewski's principle". Topological Methods in Nonlinear Analysis, 56(1), 1-30

The final published version is available online at: http://dx.doi.org/10.12775/TMNA.2019.110

Rights / License:

The terms and conditions for the reuse of this version of the manuscript are specified in the publishing policy. For all terms of use and more information see the publisher's website.

This item was downloaded from IRIS Università di Bologna (https://cris.unibo.it/)

When citing, please refer to the published version. 


\title{
CORRIGENDUM AND ADDENDUM TO "NON-AUTONOMOUS QUASILINEAR ELLIPTIC EQUATIONS AND WAŻEWSKI'S PRINCIPLE"
}

\author{
Matteo Franca
}

\begin{abstract}
In this addendum we fill a gap in a proof and we correct some results appearing in [12]. In the original paper [12] we classified positive solutions for the following equation

$$
\Delta_{p} u+K(r) u^{\sigma-1}=0
$$

where $r=|x|, x \in \mathbb{R}^{n}, n>p>1, \sigma=n p /(n-p)$ and $K(r)$ is a function strictly positive and bounded. In fact [12] had two main purposes. First, to establish asymptotic conditions which are sufficient for the existence of ground states with fast decay and to classify regular and singular solutions: these results are correct but need some non-trivial further explanations. Second to establish some computable conditions on $K$ which are sufficient to obtain multiplicity of ground states with fast decay in a non-perturbation context. Also in this case the original argument contained a flaw: here we correct the assumptions of [12] by performing a new nontrivial construction. A third purpose of this addendum is to generalize results of [12] to a slightly more general equation

$$
\Delta_{p} u+r^{\delta} K(r) u^{\sigma(\delta)-1}=0
$$

where $\delta>-p$, and $\sigma(\delta)=p(n+\delta) /(n-p)$.
\end{abstract}

2020 Mathematics Subject Classification. Primary: 35J92, 37D10; Secondary: 34C37, $34 \mathrm{C} 45$.

Key words and phrases. p-laplace equations; invariant manifold; non-smooth systems; radial solutions; ground states; Fowler transformation; Ważewski's principle.

Partially supported by the GNAMPA project "Sistemi dinamici, metodi topologici e applicazioni all'analisi nonlineare". 


\section{Introduction}

The purpose of this addendum is to complete with some explanations the analysis started in [13] and continued in [12], and to correct some errors appearing in [12] (in fact [12] is a completion of [13], even if the latter has been published afterwards).

Both [13] and [12] are concerned with the classification of positive radial solutions to

$$
\Delta_{p} u+K(r) u^{\sigma-1}=0
$$

where $\Delta_{p} u=\operatorname{div}\left(|D u|^{p-2} D u\right), p>1$, denotes the degenerate $p$-Laplace operator, $r=|x|, x \in \mathbb{R}^{n}, n>p$ and $\sigma=n p /(n-p)$ is the Sobolev critical exponent; further in this addendum, as in [13], [12], it is always assumed (without further mentioning) that $K(r)$ is uniformly continuous and $0<a<K(r)<b<\infty$ for any $r>0$.

In fact, we take the chance to extend the discussion to the following slightly more general equation

$$
\Delta_{p} u+r^{\delta} K(r)|u|^{\sigma(\delta)-1}=0
$$

where $\delta>-p$, and $\sigma(\delta)=p(n+\delta) /(n-p)$ is the critical exponent for this modified equation.

In the whole article we restrict to consider radially symmetric solutions, so in fact we reduce to consider the following singular ODE:

$$
\left(u^{\prime}\left|u^{\prime}\right|^{p-2}\right)^{\prime}+\frac{n-1}{r} u^{\prime}\left|u^{\prime}\right|^{p-2}+r^{\delta} K(r)|u|^{\sigma(\delta)-1}=0
$$

i.e. equation (1.2) of [13] and equation (1.1) of [12]. We emphasize that in several situations positive solutions inherit the symmetry of the domain, hence entire solutions to (1.1) in $\mathbb{R}^{n}$ have to be radial, e.g. if $p=2$ and $K$ is decreasing, [5], [15], or there is $R$ such that $K$ is decreasing for $r<R$ and increasing for $r>R$ [3, Theorem 1], if $p>1$ and $K$ is a constant, see [22].

Let us introduce some notations.

A solution $u(r)$ to (1.3) is called regular if $u(0)=u_{0}>0$ and singular if it is singular in the origin, that is $\lim _{r \rightarrow 0} u(r)=+\infty$ (in fact, we have $\left.\limsup u(r) r^{(n-p) / p}>0\right)$.

By a straightforward application of de l'Hopital rule and (1.3) we find that regular solutions satisfy $u^{\prime}(0)=0$ and are smooth as solutions of (1.1) if an only if $\delta>-1$.

Further, definitively positive solutions $u(r)$ have two typical behavior as $r \rightarrow$ $+\infty$ : we say that $u(r)$ has fast decay (fd for short) if $u(r) \sim r^{-(n-p) /(p-1)}$, and that it has slow decay ( $S D$ for short) /f $\lim \sup u(r) r^{(n-p) / p}>0$.

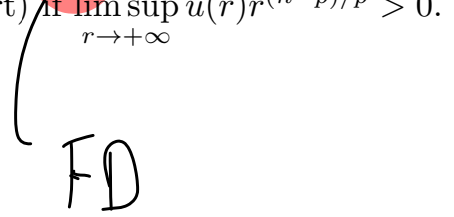


In the whole paper $u(r) \sim r^{-a}$ as $r \rightarrow \infty$ stands for

$$
0<\liminf _{r \rightarrow \infty} u(r) r^{a} \leq \limsup _{r \rightarrow \infty} u(r) r^{a}<\infty \quad \text { as } r \rightarrow \infty .
$$

We are mainly interested in positive solutions and in particular in ground states ( $G S$ for short), i.e. regular solutions which are positive for any $r \geq 0$ and $\lim _{r \rightarrow+\infty} u(r)=0$, and singular ground states ( $S G S$ for short) i.e. singular solutions which are positive for any $r>0$ and $\lim _{r \rightarrow+\infty} u(r)=0$. Further crossing solutions are solutions $u(r)$ of (1.3) such that $u(r)>0$ for $r<R$ and $u(R)=0$; they can also be regarded as Dirichlet solutions in the ball of radius $R$.

Equation (1.1) has raised a lot of interest starting from the late 80's with the classical $p=2$ case and from the 90 s in the $p>1$ case, and nowadays a lot of results are available. Most of the literature focuses on positive solutions and in particular on GS with FD, motivated by the relevance they have in applications, i.e. in differential geometry, quantum mechanics and astrophysics, especially in the classical $p=2$ case, see e.g. [6]. In fact for $p=2$ the existence of GS with FD is equivalent to the existence of a metric in $\mathbb{R}^{n}$ with scalar curvature $K(|x|)$, see e.g. [6]; further, roughly speaking, the asymptotic conditions are required in quantum mechanics and astrophysics in order to deal with physical solutions (e.g. with finite mass if we regard (1.2) as a generalized Matukuma equation, see e.g. [9]).

The purpose of this addendum, together with [13], [12], is to give conditions sufficient to obtain the existence of GS with FD and also structure results for positive solutions.

A key role in this context is played by the so called Pohozaev function (see e.g. [19]):

$$
J^{+}(r):=\int_{0}^{r} K^{\prime}(s) s^{n} d s .
$$

It is well known that GS with FD cannot exist if $J^{+}(r)$ has constant sign for $r>0$, see e.g. [10], [18]. However, when $J^{+}(r)$ changes sign, one should expect for GS with FDand in literature there are lots of papers giving several different conditions ensuring their existence. These assumptions are of two types: firstly one may require asymptotic conditions on $K$ as $r \rightarrow 0$ and as $r \rightarrow+\infty$ (usually $K$ has to be steep enough), see e.g. [2], [25], [13], [12], [9]; secondly one may ask for $K$ to be a perturbation of a constant, namely either $K(r)=1+\varepsilon k(r)$ (regular perturbation, i.e. small oscillations), see e.g. [24], [23], [16], or $K(r)=k\left(r^{\varepsilon}\right)$ (singular perturbation, i.e. slow oscillations), see e.g. [16], [1], [11], [14].

All the proofs in [12], [13] and in this addendum are performed after the change of variables $(2.1)$ of [13], (2.2) of [12], i.e.

$$
x_{1}=u(r) r^{\alpha}, \quad x_{2}=u^{\prime}(r)\left|u^{\prime}(r)\right|^{p-2} r^{\beta}, \quad r=e^{t}, \quad K\left(\mathrm{e}^{t}\right)=\phi(t),
$$


where $\alpha=(n-p) / p$ and $\beta=n(p-1) / p$ which allows to pass from (1.3) to

$$
\left\{\begin{array}{l}
\dot{x}_{1}=f_{1}(x):=\alpha x_{1}+x_{2}\left|x_{2}\right|^{(2-p) /(p-1)}, \\
\dot{x}_{2}=f_{2}(x ; t):=-\alpha x_{2}-\phi(t)\left|x_{1}\right|^{\sigma-1} .
\end{array}\right.
$$

Remark 1.1. In [13], [12] the $\delta=0$ case was considered. However, the change of variables (1.5) applies to the general case $\delta>-p$. Notice that with this particular choice of $\sigma(\delta)$ the term $\mathrm{e}^{\delta t}=r^{\delta}$ disappears; in fact the passage from $\delta=0$ to $\delta>-p$ only affects the value of $\sigma$. As we will see below (1.6) is Hamiltonian for any $\sigma$ if $\phi$ is constant, so equation (1.3) is critical. Hence the original discussion performed in [12], [13] for $\delta=0$ is easily extended to $\delta>-p$ with almost no effort.

REMARK 1.2. We think it is worthwhile to point out that if we replace the potential $K(r) r^{\delta} u^{\sigma(\delta)-1}$ with the term $K(r) r^{\delta} u^{q-1}$ then (1.3) is subcritical if $p<q<\sigma(\delta)$ and supercritical if $q>\sigma(\delta)$ : in these cases the structure of positive solutions is completely altered.

In particular if $\delta>0$ and $q=\sigma(0)=\frac{n p}{n-p}<\sigma(\delta)$ we have a subcritical situation while if $-p<\delta<0$ and $q=\sigma(0)>\sigma(\delta)$ we have a supercritical situation.

The purpose of [13] was to obtain a structure result for positive solutions when $2 n /(n+2) \leq p \leq 2$, and in particular, existence of GS with FD, requiring the following asymptotic conditions:

$\left(\alpha^{-}\right)$There is $\rho>0$ such that $K(r)$ is nonincreasing for $r \in[0, \rho)$ and $K^{\prime}(r) r^{-n /(p-1)} \notin L^{1}(0, \rho]$.

$\left(\alpha^{+}\right)$There is $\rho>0$ such that $K(r)$ is nondecreasing for $r \in[0, \rho)$ and $K^{\prime}(r) r^{-n /(p-1)} \notin L^{1}(0, \rho]$.

$\left(\Omega^{-}\right)$There is $R>0$ such that $K(r)$ is nonincreasing for $r>R$ and $K^{\prime}(r) r^{n}$ $\notin L^{1}[R,+\infty)$.

$\left(\Omega^{+}\right)$There is $R>0$ such that $K(r)$ is nondecreasing for $r>R$ and $K^{\prime}(r) r^{n}$ $\notin L^{1}[R,+\infty)$.

Paper [12] had two main purposes. The first one was to extend the results of [13] to the whole range $p>1$, i.e. to the case where (1.6) is not smooth (and local uniqueness of the solutions is not guaranteed): this is the content of all the results of $[12, \S 2]$, which are existence and classification results obtained by requiring $\left(\alpha^{ \pm}\right)$and $\left(\Omega^{ \pm}\right)$. The second one was to establish some multiplicity results in a non-perturbative setting: this is the content of $[12, \S 4]$. The purpose of this addendum is to fill a gap in the proof of the results of $[12, \S 2]$, to correct the results appearing in $[12, \S 4]$, and to extend all the results of [12] to the slightly more general equation (1.2). 
Let us begin with the results of $[12, \S 2]$. We recall in particular [12, Theorem $2.4]$ which is repeated here for convenience of the reader, already referred to the more general equation (1.3).

Theorem 1.3. Consider equation (1.3), and assume $\left(\alpha^{+}\right)$and $\left(\Omega^{-}\right)$.

(a) There exist uncountably many crossing solutions.

(b) There exist uncountably many GS with SD.

(c) There exists at least one GS with FD.

(d) There exist uncountably many Dirichlet solutions $u(r)$ in exterior domains: for each such $u(r)$ there exists $R>0$ such that $u(R)=0$ and $u(r) \sim r^{-(n-p) /(p-1)}$, as $r \rightarrow \infty$.

(e) There exist uncountably many SGS with FD.

(f) No other positive solutions can exist but the ones described and, possibly, $S G S$ with $S D$.

Then if we assume $\left(\alpha^{+}\right)$and $\Omega^{-}$and that there exists $R>0$ such that $J^{+}(r) \geq 0$ for any $r<R$ and $K^{\prime}(r) \leq 0$ for any $r>R$ we got a complete classification result for regular solutions and in particular the uniqueness of the GS with FD, and the existence of a SGS with SD, see [12, Corollary 2.5]. The proof is obtained simply putting together [12, Theorem 2.4] (i.e. Theorem 1.3 of this article) and [19, Theorem 1].

Similarly, in [12, Theorem 2.6] we proved the existence of a GS with FD when $\left(\alpha^{-}\right)$and $\left(\Omega^{+}\right)$simultaneously hold, i.e. we get the following.

Theorem 1.4. Assume $\left(\alpha^{-}\right)$and $\left(\Omega^{+}\right)$. Then (1.3) admits uncountably many crossing solutions, uncountably many solutions $u(r)$ of the Dirichlet problem in exterior domains, and at least one GS with FD.

Notice that in the setting of Theorem 1.4 in general we cannot expect for uniqueness of GS with FD and we do not get a structure result for positive solutions. In fact in the Laplace case there are some perturbative results which obtain multiplicity of the GS with FD requiring a unique change of sign of the function $J^{+}(r)$, see [6], [23], [11].

Even if Theorems 1.3, 1.4 and all the results of $[12, \S 2]$ are correct, there is a gap in the original proof in [12]: a first purpose of this addendum is to fill this gap.

The main problem in the original argument is caused by a missing assumption in [12, Theorem 3.3], where we have constructed the stable and the unstable sets $\widehat{\boldsymbol{W}}^{\boldsymbol{s}}(\tau), \widehat{\boldsymbol{W}}^{\boldsymbol{u}}(\tau)$ of (1.6), which corresponds respectively to the fast decay and the regular solutions of (1.3).

Namely to fix the proof of [12, Theorem 3.3] we need to assume either of the following:

(H1) $b / a<n /(n-p)$, where $0<a<K(r)<b$ for any $r>0$, 
(H2) $\lim _{r \rightarrow 0} K(r)=K(0) \in(0,+\infty)$ and $\lim _{r \rightarrow+\infty} K(r)=K(\infty) \in(0,+\infty)$.

The argument performed in the proof of [12, Theorem 3.3] is correct if we add assumption (H1). However [12, Theorem 3.3] can be proved also replacing (H1) by the more flexible (H2) but we need to perform a truncation argument. This explanation is the object of Section 3 of this addendum.

We emphasize that (H2) is always implicitly assumed if we are in the setting of Theorems 1.3, 1.4, and of all the results of $[12, \S 2]$, so the statements of these results are correct as they are, and need no changes (but the original proof were incomplete).

Further, in order to obtain an intersection between $\widehat{\boldsymbol{W}}^{\boldsymbol{u}}\left(\tau^{*}\right)$ and $\widehat{\boldsymbol{W}}^{\boldsymbol{s}}\left(\tau^{*}\right)$ for a suitable $\tau^{*} \in \mathbb{R}$ (which immediately implies the existence of a GS with FD, sēe Lemma 4.1), we simply used a continuity argument. However, especially if (H2) is replaced by $(\mathrm{H} 2)$, such an explanation fails and we need a more difficult proof: this is the object of Lemma 4.3 which is proved in Section 4.2.

Now we turn to consider $[12, \S 4]$ : in literature nowadays several results ensuring multiplicity of GS with FD are available. For the classical Laplace case we wish to mention in particular [16], [24] which requires the presence of several critical points, and [6], [23], [11] which requires the presence of a minimum, but always in a perturbative context. As far as we are aware [14] is the first paper to obtain multiplicity in a $p$-Laplace setting, but again in a perturbative setting.

The second purpose of [12] was to prove the multiplicity of GS with FD when $K$ has many critical points, but in a non-perturbative setting, thus unifying the singular and the regular perturbation results obtained in [14], i.e. [14, Theorem 3.2] and [14, Theorem 5.2]; this is done in [12, §4]. More precisely, we have constructed some computable functions $F_{u}^{+}(\tau), F_{s}^{+}(\tau), F_{u}^{-}(\tau), F_{s}^{-}(\tau)$ depending only on $K$, which allow to establish explicit conditions which guarantee the existence of multiple GS with FD. These results shed some light on the role played by the "smallness" and "slowness" conditions on the oscillations of $K$ in [14, Theorem 3.2] and [14, Theorem 5.2] and by their interplay.

However, the original result contains a flaw: we always need to assume $(\mathrm{H} 1)$ and we need to replace the original auxiliary functions $F_{u, s}^{ \pm}(\tau)$ by some more complicated ones. In fact we have the following.

TheOrem 1.5. Assame $[\mathrm{H} 1)$ and replace the original functions $F_{u}^{+}(\tau)$, $F_{s}^{+}(\tau), F_{u}^{-}(\tau), F_{s}^{-}(\tau)$ by the ones of Section 4.1 of this addendum, see (4.3)(4.6), (4.15), (4.14). Then all the results of [12, §4] (namely [12, Theorem 4.1], [12, Theorem 4.3], [12, Theorem 4.4]) hold true.

The second purpose of this addendum is to perform such a correction and to construct these new auxiliary functions $F_{u, s}^{ \pm}(\tau)$. In fact, the new argument 
is non-trivial and needs some rather careful computations: it is performed in Section 4.1.

Finally, a minor purpose of this paper is the following result.

Proposition 1.6. All the results of $[12, \S 2]$, and Theorem 1.5 of this addendum hold true for the more general equation (1.3) with $\delta>-p$.

We will refer several times to [12] and we will maintain the notation as much as possible, to help the reader.

Summing up the contribution of the present article are the following.

- We complete the proof of $[12$, Theorems $2.3,2.4]$, i.e. Theorems 1.3 and 1.4 of the present article. For this purpose we correct the construction of the sets $\widehat{\boldsymbol{W}}^{\boldsymbol{s}}(\tau), \widehat{\boldsymbol{W}}^{\boldsymbol{u}}(\tau)$ (this is the content of Theorems 3.2 and 3.5 of the present article). Further we also correct the proof of the existence of an intersection between $\widehat{\boldsymbol{W}}^{s}(\tau)$ and $\widehat{\boldsymbol{W}}^{\boldsymbol{u}}(\tau)$. In fact in [12] it is based on a simple connection argument, but it is incomplete: here it is replaced by a more articulated topological idea (see Lemma 4.3 of this article).

- We replace the asymptotic estimates of [12, Proposition 2.1] by Proposition 2.4 of the present article.

- We correct the multiplicity results appearing in $[12, \S 4]$, see Theorem 1.5 of the present article.

- We extend all the results of [13], [12] to the slightly more general equation (1.3) where $\delta>-p$.

The paper is divided as follows: in Section 2 we introduce some sets and we recall the notation which are actually used in Sections 3 and $\S 4$ to prove the main results. In Section 3 we complete and correct [12, Theorem 3.3], i.e. the construction of the connected stable and unstable sets $\widehat{\boldsymbol{W}}^{s}(\tau), \widehat{\boldsymbol{W}}^{\boldsymbol{u}}(\tau)$. In Section 4.1 we build up new sub and super-solutions which allow to construct new functions $F_{u, s}^{ \pm}(\tau)$, and to correct the results of $[12, \S 4]$. Finally, in Section 4.2 we prove the technical Lemma 4.3.

\section{Preliminaries and notation}

In this section we recall some notations introduced at the beginning of $\S 3$ in [12] and we give some new ones, then we develop the preliminary constructions needed in the next sections. The starting point of our discussion is the Fowler transformation i.e. the change of variables (1.5) which allows to pass from (1.3) to (1.6); this tool was generalized to the $p$-Laplacian by Bidaut-Veron in [4]. In fact, all the analysis will be carried on system (1.6), and will profit of phase plane techniques. 
Following [12] we denote by

$$
\boldsymbol{x}(\tau, \boldsymbol{Q} ; t)=\left(x_{1}(\tau, \boldsymbol{Q} ; t), x_{2}(\tau, \boldsymbol{Q} ; t)\right)
$$

[12] the trajectory of (1.6) which is in $\boldsymbol{Q}$ at $t=\tau$, so that $\boldsymbol{x}(\tau, \boldsymbol{Q} ; \tau)=\boldsymbol{Q}$. Sometimes [12] $(\tau, \mathcal{Q} ; \nvdash)$ we will write simply $\boldsymbol{x}(t)=\left(x_{1}(t), x_{2}(t)\right)$ leaving the dependence on $\tau$ and $\boldsymbol{Q}$

REMARK 2.1. We stress that in [12] we have denoted by $\boldsymbol{x}(\tau, \boldsymbol{Q} ; t)$ the solution of a shifted system and the notation of this article and of [12] differ slightly. So, if $\boldsymbol{x}^{[\mathbf{1 4}]}(\tau, \boldsymbol{Q} ; t)$ is the notation used in [12] and $\boldsymbol{x}(\tau, \boldsymbol{Q} ; t)$ is the notation of the present article we have

\section{$\boldsymbol{x}^{[\mathbf{1 4}]}(\tau, \boldsymbol{Q} ; t)=\boldsymbol{x}(\tau, \boldsymbol{Q} ; t+\tau), \quad$ for any $t \in \mathbb{R}$.}

We recall the definition of several sets borrowed from [12]: we invite the reader to control them on Figure 1.

$$
\begin{aligned}
\boldsymbol{U}^{+} & :=\left\{\boldsymbol{x} \mid x_{1} \geq 0, x_{2} \leq 0 \text { and } \dot{x}_{1}>0\right\} \\
\boldsymbol{U}^{-} & :=\left\{\boldsymbol{x} \mid x_{1} \geq 0, x_{2} \leq 0 \text { and } \dot{x}_{1}<0\right\}, \\
\boldsymbol{\ell} & :=\left\{\boldsymbol{x} \mid x_{1} \geq 0, x_{2} \leq 0 \text { and } \dot{x}_{1}=0\right\} .
\end{aligned}
$$

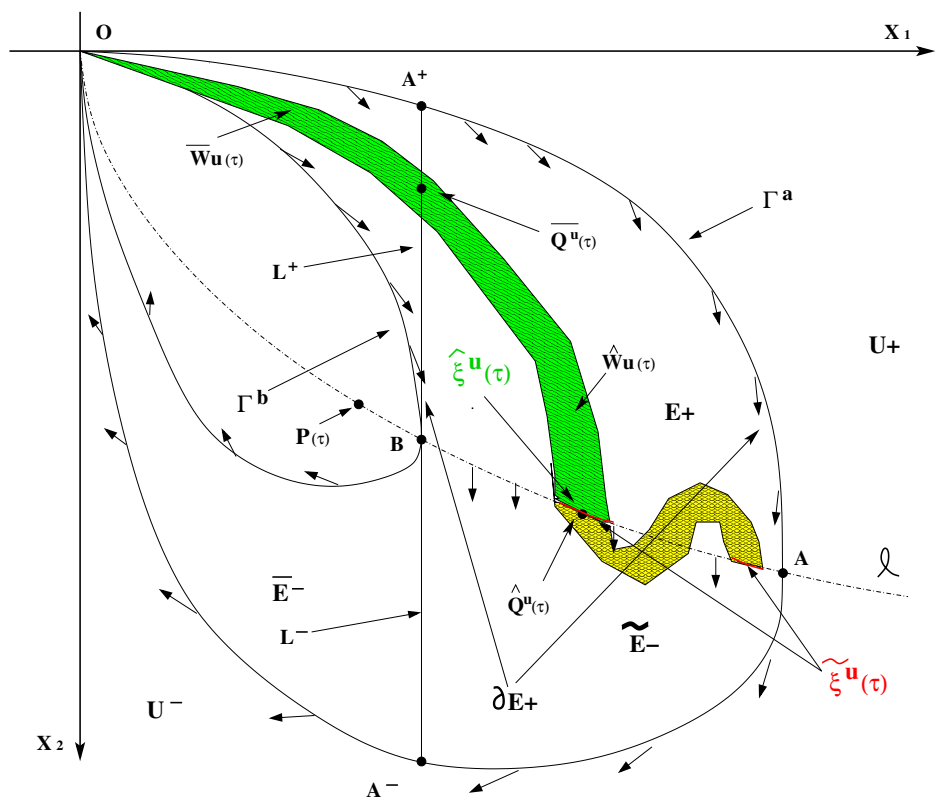

FiguRE 1. In this figure we collect several sets useful to construct the unstable sets $\widetilde{\boldsymbol{W}}^{\boldsymbol{u}}(\tau)$, its connected component $\widehat{\boldsymbol{W}}^{\boldsymbol{u}}(\tau)$, and their intersections with $\widehat{\boldsymbol{\ell}}$, i.e. $\widetilde{\boldsymbol{\xi}}^{\boldsymbol{u}}(\tau)$ and $\widehat{\boldsymbol{\xi}}^{\boldsymbol{u}}(\tau)$. The arrows indicates the direction of the flow on $\partial E$. 
One important tool in our analysis is the following energy function:

$$
H(\boldsymbol{x} ; t):=\alpha x_{1} x_{2}+\frac{p-1}{p}\left|x_{2}\right|^{p /(p-1)}+\phi(t) \frac{\left|x_{1}\right|^{\sigma}}{\sigma}
$$

Observe that by differentiating along the trajectories we get

$$
\frac{d}{d t} H(\boldsymbol{x}(t), t)=\frac{\left|x_{1}(t)\right|^{\sigma}}{\sigma} \frac{d}{d t} \phi(t) .
$$

Notice that (2.2) gives a dynamical interpretation of the Pohozaev identity. When $\phi(t) \equiv \mathcal{K}$, then (1.6) is autonomous and $H$ is a first integral, so we can draw each trajectory of (1.6). We denote by $Z^{\mathcal{K}}(t)$ the homoclinic trajectory of the frozen system (1.6) where $\phi(t) \equiv \mathcal{K}$, contained in $x_{1} \geq 0$ and such that $\boldsymbol{Z}^{\mathcal{K}}(0) \in \boldsymbol{\ell}$; we set $\boldsymbol{\Gamma}^{\mathcal{K}}:=\left\{\boldsymbol{Z}^{\mathcal{K}}(t) \mid t \in \mathbb{R}\right\}$. Fix $\tau \in \mathbb{R}$; we stress that $\boldsymbol{\Gamma}^{\phi(\tau)}$ is easily obtained as part of the 0 -level set of the function $H(\boldsymbol{x} ; \tau)$.

A key point in the whole analysis is that $Z^{\mathcal{K}}(t)=\left(U^{\mathcal{K}}(t), V^{\mathcal{K}}(t)\right)$ is explicitly known; in particular, we have (cf. [21, §1])

$$
\begin{aligned}
U^{\mathcal{K}}(t)=\left\{D_{1}\left[\mathrm{e}^{-t(p+\delta) / p}+(p-1) \mathrm{e}^{(p+\delta t /(p(p-1))}\right]\right. & \}^{-(n-p) /(p+\delta)} \\
& \times\left(D_{2} \mathcal{K}\right)^{-(n-p) /(p(p+\delta))}
\end{aligned}
$$

where

$$
D_{1}=(p-1)^{-(p-1) / p} \quad \text { and } \quad D_{2}=\frac{1}{n+\delta}\left[\frac{p-1}{n-p}\right]^{p-1} .
$$

We recall that in the whole addendum there are positive constants $a, b$ such that $a<K(r)<b$ for any $r \geq 0$. Notice that $\Gamma^{b}$ lies in the compact set enclosed by $\Gamma^{\boldsymbol{a}}$. We denote by $\boldsymbol{E}$ the compact set enclosed by $\boldsymbol{\Gamma}^{\boldsymbol{a}}, \boldsymbol{\Gamma}^{\boldsymbol{b}}$ and the origin. We denote by $\boldsymbol{\partial} \boldsymbol{E}=\boldsymbol{\Gamma}^{\boldsymbol{a}} \cup \boldsymbol{\Gamma}^{\boldsymbol{b}}$, by $\boldsymbol{E}^{ \pm}=\boldsymbol{E} \cap\left(\boldsymbol{U}^{ \pm} \cup \boldsymbol{\ell}\right)$ and by $\boldsymbol{\partial} \boldsymbol{E}^{ \pm}=\boldsymbol{\partial} \boldsymbol{E} \cap\left(\boldsymbol{U}^{ \pm} \cup \boldsymbol{\ell}\right)$. Let $\boldsymbol{A}=\left(A_{1}, A_{2}\right), \boldsymbol{B}=\left(B_{1}, B_{2}\right)$ be the points of intersection $\{\boldsymbol{A}\}:=\left(\boldsymbol{\Gamma}^{\boldsymbol{a}} \cap \boldsymbol{\ell}\right)$ and $\{\boldsymbol{B}\}:=\left(\boldsymbol{\Gamma}^{\boldsymbol{b}} \cap \boldsymbol{\ell}\right)$. We denote by $\widehat{\boldsymbol{\ell}}$, the branch of $\boldsymbol{\ell}$ between $\boldsymbol{B}$ and $\boldsymbol{A}$ (without endpoints).

We observe that the "frozen" autonomous system (1.6) where $\phi(t) \equiv \mathcal{K}$ admits a unique critical point in $x_{1}>0$, and it will be denoted by $\boldsymbol{P}(\mathcal{K})=$ $\left(P_{1}(\mathcal{K}), P_{2}(\mathcal{K})\right)$. We collect here the numerical values of some constants that will be of use in the whole article

$$
\begin{aligned}
P_{1}(\mathcal{K}) & :=\left(\frac{\alpha^{p}}{\mathcal{K}}\right)^{1 /(\sigma-p)}, & P_{2}(\mathcal{K}) & =-\left[\alpha P_{1}(\mathcal{K})\right]^{p-1}, \\
B_{1} & :=\left(\frac{\sigma \alpha^{p}}{p b}\right)^{1 /(\sigma-p)}, & B_{2} & =-\left[\alpha B_{1}\right]^{p-1}, \\
A_{1} & :=\left(\frac{\sigma \alpha^{p}}{p a}\right)^{1 /(\sigma-p)}, & A_{2} & =-\left[\alpha A_{1}\right]^{p-1} .
\end{aligned}
$$

From elementary phase plane arguments we get the following lemma. 
Lemma 2.2. Assume $0<a<K(r)<b<\infty$ then the flow of (1.6) on $\boldsymbol{\partial} \boldsymbol{E}^{+} \backslash\{\boldsymbol{A}, \boldsymbol{B}\}$ points towards the interior of $\boldsymbol{E}^{+}$and on $\boldsymbol{\partial} \boldsymbol{E}^{-} \backslash\{\boldsymbol{A}, \boldsymbol{B}\}$ points towards the exterior of $\boldsymbol{E}^{-}$.

Proof. The proof is borrowed from [12], we repeat it here for convenience of the reader. We just consider the case of $\boldsymbol{\partial} \boldsymbol{E}^{+}$, the case of $\boldsymbol{\partial} \boldsymbol{E}^{-}$is analogous. Let $\boldsymbol{x}^{\boldsymbol{a}}(t):=\boldsymbol{x}^{\boldsymbol{a}}(\tau, \boldsymbol{Q} ; t)$ and $\boldsymbol{x}^{\boldsymbol{b}}(t):=\boldsymbol{x}^{\boldsymbol{b}}(\tau, \boldsymbol{R} ; t)$ be trajectories of (1.6) where $K \equiv a$ and $K \equiv b$ respectively and $\boldsymbol{Q} \in\left(\boldsymbol{\Gamma}^{\boldsymbol{a}} \cap \boldsymbol{U}^{+}\right), \boldsymbol{R} \in\left(\boldsymbol{\Gamma}^{\boldsymbol{b}} \cap \boldsymbol{U}^{+}\right)$.

Notice that $\boldsymbol{x}^{\boldsymbol{a}}(\tau)=\boldsymbol{Q}=\boldsymbol{x}(\tau, \boldsymbol{Q} ; \tau)$ and $\boldsymbol{x}^{\boldsymbol{b}}(\tau)=\boldsymbol{R}=\boldsymbol{x}(\tau, \boldsymbol{R} ; \tau)$, hence $\dot{x}_{1}^{a}(\tau)=\dot{x}_{1}(\tau, \boldsymbol{Q} ; \tau), \dot{x}_{1}^{b}(\tau)=\dot{x}_{1}(\tau, \boldsymbol{R} ; \tau)$, but

$$
\dot{x}_{2}(\tau, \boldsymbol{Q} ; \tau)<\dot{x}_{2}^{a}(\tau), \quad \dot{x}_{2}(\tau, \boldsymbol{R} ; \tau)>\dot{x}_{2}^{b}(\tau) .
$$

Then the lemma follows from elementary phase portrait considerations recalling that, by construction, $\dot{\boldsymbol{x}}^{\boldsymbol{a}}(\tau)$ is tangent to $\boldsymbol{\Gamma}^{\boldsymbol{a}}$ in $\boldsymbol{Q}$ while $\dot{\boldsymbol{x}}^{\boldsymbol{b}}(\tau)$ is tangent to $\boldsymbol{\Gamma}^{\boldsymbol{b}}$ in $\boldsymbol{R}$.

A key role in the whole analysis is played by the following sets, constructed below via Lemma 2.2 and Ważewski's principle.

$$
\begin{aligned}
& \widetilde{\boldsymbol{W}}^{\boldsymbol{s}}(\tau):=\left\{\boldsymbol{Q} \mid \boldsymbol{x}(\tau, \boldsymbol{Q} ; t) \in \boldsymbol{E}^{-} \text {for any } t \geq \tau\right\}, \\
& \widetilde{\boldsymbol{W}^{\boldsymbol{u}}}(\tau):=\left\{\boldsymbol{Q} \mid \boldsymbol{x}(\tau, \boldsymbol{Q} ; t) \in \boldsymbol{E}^{+} \text {for any } t \leq \tau\right\} .
\end{aligned}
$$

Since (1.6) contains no invariant sets in $\boldsymbol{E}$ we easily get the following, see $\S 2$ in $[12]$.

REMARK 2.3. Let $\boldsymbol{x}(t)$ be a solution of (1.6). If $\boldsymbol{x}(t) \rightarrow(0,0)$ as $t \rightarrow-\infty$ and $x_{1}(t)>0$ definitively, then there is $\tau \in \mathbb{R}$ such that $\boldsymbol{x}(\tau)=\boldsymbol{Q} \in \widetilde{\boldsymbol{W}}^{\boldsymbol{u}}(\tau)$ and $\boldsymbol{x}(t) \in \boldsymbol{E}^{+}$for any $t \leq \tau$. Viceversa, if $\boldsymbol{Q} \in \widetilde{\boldsymbol{W}}^{\boldsymbol{u}}(\tau)$ then $\boldsymbol{x}(\tau, \boldsymbol{Q} ; t) \in \boldsymbol{E}^{+}$ for any $t \leq \tau$ and $\lim _{t \rightarrow-\infty} \boldsymbol{x}(\tau, \boldsymbol{Q} ; t)=(0,0)$.

Analogously, if $\boldsymbol{x}(t) \rightarrow(0,0)$ as $t \rightarrow+\infty$ and $x_{1}(t)>0$ definitively, then there is $\tau \in \mathbb{R}$ such that $\boldsymbol{x}(\tau)=\boldsymbol{Q} \in \widetilde{\boldsymbol{W}}^{\boldsymbol{s}}(\tau)$ and $\boldsymbol{x}(t) \in \boldsymbol{E}^{-}$for any $t \geq \tau$. Viceversa, if $\boldsymbol{Q} \in \widetilde{\boldsymbol{W}}^{\boldsymbol{s}}(\tau)$ then $\boldsymbol{x}(\tau, \boldsymbol{Q} ; t) \in \boldsymbol{E}^{-}$for any $t \geq \tau$ and $\lim _{t \rightarrow+\infty} \boldsymbol{x}(\tau, \boldsymbol{Q} ; t)=(0,0)$.

If system (1.6) is smooth, i.e if $1<p \leq 2$ and $\sigma \geq 2, \widetilde{\boldsymbol{W}}^{\boldsymbol{s}}(\tau)$ and $\widetilde{\boldsymbol{W}}^{\boldsymbol{u}}(\tau)$ are subsets of the stable and the unstable leaves of the origin, hence they are 1 dimensional immersed manifolds, see e.g. [8, §13]. In the general setting $1<$ $p<\sigma(1.6)$ is just Hölder, and we are just able to show that $\widetilde{\boldsymbol{W}}^{\boldsymbol{s}}(\tau)$ and $\widetilde{\boldsymbol{W}}^{\boldsymbol{u}}(\tau)$ are non-empty, contain compact and connected sets, i.e. continua, denoted by $\widehat{\boldsymbol{W}}^{\boldsymbol{s}}(\tau), \widehat{\boldsymbol{W}}^{\boldsymbol{u}}(\tau)$ which contain the origin and intersect $\widehat{\boldsymbol{\ell}}$, see $\S 3$. We recall that if $\mathcal{C}$ is a continuum, then, for any two points $\boldsymbol{Q} \neq \boldsymbol{R}$ in $\mathcal{C}$ and any $\varepsilon>0$ there is a path $\gamma$ joining $\boldsymbol{Q}$ with $\boldsymbol{R}$ and contained in a $\varepsilon$-neighbourhood of $\mathcal{C}$, see [20, p. 345].

There are some basic relations between the solution $u(r)$ of (1.3) and the corresponding solution $\boldsymbol{x}(t)$ of (1.6). Obviously $u^{\prime}(r)<0<u(r)$ if and only if 
$x_{2}(t)<0<x_{1}(t)$ when $r=\mathrm{e}^{t}$. Further we have the following results, borrowed from [13, Proposition 2.6], [13, Remark 2.7].

Proposition 2.4. Consider a solution $u(r)$ of (1.3) and the corresponding trajectory $\boldsymbol{x}(t)$ of (1.6). If $u(r)>0$ for $r$ small it has one of these possible behaviors as $r \rightarrow 0$ :

(a) $\lim _{t \rightarrow-\infty} \boldsymbol{x}(t)=(0,0)\left(\right.$ in particular $\left.\lim _{t \rightarrow-\infty} H(\boldsymbol{x}(t), t)=0\right)$, hence $u(r)$ is regular.

(b) $\lim _{t \rightarrow-\infty} H(\boldsymbol{x}(t), t)<0$, hence $u(r) \sim r^{-\alpha}$ as $r \rightarrow 0$; in particular $u(r)$ is singular.

(c) $\lim _{t \rightarrow-\infty} H(\boldsymbol{x}(t), t)=0$, and $\boldsymbol{x}(t) \not \rightarrow(0,0)$, hence

$$
\lim _{r \rightarrow 0} u(r)=+\infty, \quad 0=\liminf _{r \rightarrow 0} u(r) r^{\alpha}<\limsup _{r \rightarrow 0} u(r) r^{\alpha}<\infty ;
$$

in particular $u(r)$ is singular.

Analogously, if $u(r)>0$ for $r$ large it has one of these possible behaviors as $r \rightarrow+\infty$ :

(d) $\lim _{t \rightarrow+\infty} \boldsymbol{x}(t)=(0,0)$ (in particular $\lim _{t \rightarrow+\infty} H(\boldsymbol{x}(t), t)=0$ ), hence $u(r)$ has $F D$.

(e) $\lim _{t \rightarrow+\infty} H(\boldsymbol{x}(t), t)<0$, hence $u(r) \sim r^{-\alpha}$ as $r \rightarrow+\infty$; in particular $u(r)$ has $S D$.

(f) $\lim _{t \rightarrow+\infty} H(\boldsymbol{x}(t), t)=0$, and $\boldsymbol{x}(t) \nrightarrow \rightarrow(0,0)$, hence

$$
\lim _{r \rightarrow+\infty} u(r)=+\infty, \quad 0=\liminf _{r \rightarrow+\infty} u(r) r^{\alpha}<\limsup _{r \rightarrow+\infty} u(r) r^{\alpha}<+\infty ;
$$

in particular $u(r)$ has $S D$.

In [12, Proposition 2.1] we missed case (c) both as $r \rightarrow 0$ and as $r \rightarrow+\infty$.

In [7, Theorem 1.6] Chen and Lin showed that if $p=2, \lim _{r \rightarrow 0} K(r)=K(0)>0$, $K^{\prime}(r)<0$ and $K$ is steep enough (i.e. $K^{\prime}(r) \sim r^{a}$ where $0<a<\alpha-1$ ) then (1.6) admits trajectories $\boldsymbol{x}(t)$ having the whole $\boldsymbol{\partial} \boldsymbol{E}^{K(0)}$ as $\alpha$-limit set, i.e. $u(r)$ has behaviour (c) as $r \rightarrow 0$. However if $p=2, \lim _{r \rightarrow 0} K(r)=K(0)>0, K^{\prime}(r)<0$ and $K$ is flat enough (i.e. $K^{\prime}(r) \sim r^{a}$ where $a>\alpha-1$ ) case (c) cannot take place when $r \rightarrow 0$, see [7, Theorem 1.4]. A specular situation is found for $r$ large.

Our goal will be to obtain the existence of intersections between $\widetilde{\boldsymbol{W}}^{\boldsymbol{u}}(\tau)$ and $\widetilde{\boldsymbol{W}}^{\boldsymbol{s}}(\tau)$ and to show via Proposition 2.4 that the corresponding solutions $u(r)$ of (1.3) are GS with FD. The key idea of both [13], [12] and of this addendum is to use the function $H$ and some sub and super-solutions, built from the autonomous system where $K \equiv a$ or $K \equiv b$, to evaluate the mutual positions of $\widetilde{\boldsymbol{W}}^{\boldsymbol{u}}(\tau)$ and $\widetilde{\boldsymbol{W}}^{\boldsymbol{s}}(\tau)$. 


\section{Construction of stable and unstable sets: corrigendum and addendum to [12, Section 3$]$.}

This subsection is devoted to the completion and correction of [12, Theorem 3.3], i.e. the results concerning the existence and the properties of $\widetilde{\boldsymbol{W}}^{\boldsymbol{u}}(\tau)$ and $\widetilde{\boldsymbol{W}}^{\boldsymbol{s}}(\tau)$. In particular, we aim to show that for any $\tau \in \mathbb{R}, \widetilde{\boldsymbol{W}}^{\boldsymbol{u}}(\tau)$ (respectively, $\left.\widetilde{\boldsymbol{W}}^{\boldsymbol{s}}(\tau)\right)$ contains a compact connected subset, denoted by $\widehat{\boldsymbol{W}}^{\boldsymbol{u}}(\tau)$ (respectively, $\widehat{\boldsymbol{W}}^{\boldsymbol{s}}(\tau)$ ) which contains the origin and intersects $\widehat{\boldsymbol{\ell}}$.

The construction of the stable and the unstable sets are based on some simple geometrical ideas.

Lemma 3.1. Assume (H1) then the flow of (1.6) on $\widehat{\boldsymbol{\ell}}$ points towards $\boldsymbol{U}^{-}$.

Proof. Let $\boldsymbol{Q}=\left(Q_{1}, Q_{2}\right) \in \widehat{\boldsymbol{\ell}}$ and consider $\boldsymbol{x}(\tau, \boldsymbol{Q} ; t)$; observe that

$$
\dot{x}_{1}(\tau, \boldsymbol{Q} ; t)=0 .
$$

Further

$$
f_{2}(\boldsymbol{Q}, t)=Q_{1}^{p-1}\left[\alpha^{p}-\phi(t) Q_{1}^{\sigma-p}\right] \leq Q_{1}^{p-1}\left[\alpha^{p}-a B_{1}^{\sigma-p}\right]<0
$$

where in the last inequality we used (H1); so Lemma 3.1 follows. Notice that (H1) is equivalent to require that $\boldsymbol{P}(\phi(\tau))$ is on the left of $\boldsymbol{B}$ (hence of the whole $\widehat{\ell}$ ) for any $\tau \in \mathbb{R}$.

Then, repeating the argument of [12, Section 3] without any change, we obtain the following weaker version of [12, Theorem 3.3] (and correspondingly of [13, Lemma 2.8]).

Theorem 3.2. Assume (H1). Then, for any $\tau$, the sets $\widetilde{\boldsymbol{W}}^{\boldsymbol{s}}(\tau)$ (correspondingly the sets $\left.\widetilde{\boldsymbol{W}}^{\boldsymbol{u}}(\tau)\right)$ contain compact and connected continua $\widehat{\boldsymbol{W}}^{\boldsymbol{s}}(\tau)$ (correspondingly $\left.\widehat{\boldsymbol{W}}^{u}(\tau)\right)$ which contain the origin and a point in $\widehat{\boldsymbol{\ell}}$.

The construction of $\widehat{\boldsymbol{W}}^{s}(\tau)$ and $\widehat{\boldsymbol{W}}^{\boldsymbol{u}}(\tau)$ relies on the following topological Lemma, borrowed from [20, Lemma 4], see also [12, Lemma 3.2].

Lemma 3.3. Let $\mathcal{R}$ be a closed set homeomorphic to a full triangle. We call $\boldsymbol{O}, \boldsymbol{A}$ and $\boldsymbol{B}$ the vertices and $\boldsymbol{o}, \boldsymbol{a}, \boldsymbol{b}$ the edges which are opposite to the respective vertex. Let $\mathcal{S} \subset \mathcal{R}$ be a closed set such that $\boldsymbol{\gamma} \cap \mathcal{S} \neq \emptyset$, for any path $\boldsymbol{\gamma} \subset \mathcal{R}$ joining $\boldsymbol{a}$ with $\boldsymbol{b}$. Then $\mathcal{S}$ contains a closed connected set which contains $\boldsymbol{O}$ and at least one point of $\boldsymbol{o}$.

Proof of Theorem 3.2. We just sketch the argument for convenience of the reader remanding to [12, Theorem 3.3] for a full fledged proof.

We develop the proof just for $\widehat{\boldsymbol{W}}^{s}(\tau)$ the case of $\widehat{\boldsymbol{W}}^{\boldsymbol{u}}(\tau)$ is analogous. Fix $\tau \in \mathbb{R}$, for any point $\boldsymbol{Q} \in \boldsymbol{E}^{-}$we define the smallest time $T(\boldsymbol{Q}) \geq \tau$ such that $\boldsymbol{x}(\tau, \boldsymbol{Q} ; T) \in \boldsymbol{\partial} \boldsymbol{E}^{-}$, and we set $T(\boldsymbol{Q})=+\infty$ if $\boldsymbol{x}(\tau, \boldsymbol{Q} ; t) \in \boldsymbol{E}^{-}$, for any 
$t \geq \tau$. Since the flow of (1.6) on $\boldsymbol{\partial} \boldsymbol{E}^{-}$is transversal we can show that the set $\widehat{\boldsymbol{E}}^{-}:=\left\{\boldsymbol{Q} \in \boldsymbol{E}^{-} \mid T(\boldsymbol{Q})<\infty\right\}$ is relatively open in $\boldsymbol{E}^{-}$, and that the exit time $T: \widehat{\boldsymbol{E}}^{-} \rightarrow[\tau,+\infty)$ is continuous. Therefore the function $C(\boldsymbol{Q}): \widehat{\boldsymbol{E}}^{-} \rightarrow \boldsymbol{\partial} \boldsymbol{E}^{-}$ defined by $C(\boldsymbol{Q})=\boldsymbol{x}(\tau, \boldsymbol{Q} ; T(\boldsymbol{Q}))$ is continuous too. Let us denote by $\mathfrak{A}:=$ $C^{-1}\left(\boldsymbol{\Gamma}^{a} \cap \boldsymbol{E}^{-}\right)$and by $\mathfrak{B}:=C^{-1}\left(\boldsymbol{\Gamma}^{\boldsymbol{b}} \cap \boldsymbol{E}^{-}\right)$; recalling that $\{(0,0)\} \notin\left(\boldsymbol{\Gamma}^{a} \cup \boldsymbol{\Gamma}^{b}\right)$, from the continuity of $C$ we easily get that $\mathfrak{A}$ and $\mathfrak{B}$ are open in $\boldsymbol{E}^{-}$. Further $\left(\Gamma^{a} \cap U^{-}\right) \in \mathfrak{A},\left(\Gamma^{b} \cap \boldsymbol{U}^{-}\right) \in \mathfrak{B}$ and in particular $\boldsymbol{A} \in \mathfrak{A}, \boldsymbol{B} \in \mathfrak{B}$.

Notice now that $\left[\boldsymbol{E}^{-} \backslash(\mathfrak{A} \cup \mathfrak{B})\right]=\widetilde{\boldsymbol{W}}^{\boldsymbol{s}}(\tau)$ since the flow on $\hat{\boldsymbol{\ell}}$ points towards $\boldsymbol{U}^{-}$, see also Remark 2.3. Hence $\widetilde{\boldsymbol{W}}^{\boldsymbol{s}}(\tau)$ is closed. From a connection argument it follows that there is $\boldsymbol{Q} \in \widehat{\boldsymbol{\ell}}, \boldsymbol{Q} \notin(\boldsymbol{A} \cup \mathfrak{B})$, i.e. $\boldsymbol{Q} \in \widetilde{\boldsymbol{W}}^{\boldsymbol{s}}(\tau)$ so $\widetilde{\boldsymbol{W}}^{\boldsymbol{s}}(\tau) \neq \emptyset$. Further, for any path $\boldsymbol{\gamma}:[0,1] \rightarrow \boldsymbol{E}^{-}$such that $\gamma(0) \in\left[\boldsymbol{\Gamma}^{\boldsymbol{a}} \cap \boldsymbol{E}^{-}\right]$ and $\boldsymbol{\gamma}(1) \in\left[\boldsymbol{\Gamma}^{\boldsymbol{b}} \cap \boldsymbol{E}^{-}\right]$, there is $s \in(0,1)$ such that $\gamma(s) \notin(\boldsymbol{A} \cup \mathfrak{B})$ again from a connection argument. Hence $\gamma(s) \in \widetilde{\boldsymbol{W}}^{s}(\tau)$. So, we can apply Lemma 3.3, where $\mathcal{R}=\boldsymbol{E}^{-}, \mathcal{S}=\widetilde{\boldsymbol{W}}^{s}(\tau)$ and get the existence of the set $\widehat{\boldsymbol{W}}^{s}(\tau)$ with the desired properties.

Remark 3.4. We emphasize that in the original proof of [12, Section 3] we did not ask for (H1). Consequently the flow of (1.6) on $\boldsymbol{\partial} \boldsymbol{E}^{-}$pointed towards the exterior of $\boldsymbol{E}^{-}$, cf. Lemma 2.2, but it could be not transversal on $\widehat{\boldsymbol{\ell}}$. Hence, without requiring (H1), it might happen that for some $Q \in \widehat{\ell}$ the trajectory $\boldsymbol{x}(\tau, \boldsymbol{Q} ; t)$ does not enter $\boldsymbol{E}^{-}$for $t$ in a right neighbourhood of $\tau$ and the argument of the proof of Theorem 3.2 (i.e. [12, Theorem 3.3]) fails.

Now we proceed to prove Theorem 3.2 but asking for (H2) instead of (H1), i.e. we aim to prove the following.

THEOREM 3.5. Assume (H2), then for any $\tau$ the sets $\widetilde{\boldsymbol{W}}^{\boldsymbol{s}}(\tau)$ (correspondingly the sets $\widetilde{\boldsymbol{W}}^{\boldsymbol{u}}(\tau)$ ) contain compact and connected continua $\widehat{\boldsymbol{W}}^{\boldsymbol{s}}(\tau)$ (correspondingly $\left.\widehat{\boldsymbol{W}}^{\boldsymbol{u}}(\tau)\right)$ which contain the origin and a point in $\widehat{\boldsymbol{\ell}}$.

Once more we recall that (H2) is always satisfied if we require one between $\left(\alpha^{-}\right)$and $\left(\alpha^{+}\right)$and one between $\left(\Omega^{-}\right)$and $\left(\Omega^{+}\right)$. The proof of Theorem 3.5 is based on some geometrical constructions and on a truncation argument.

We develop the whole idea just for the stable set $\widetilde{\boldsymbol{W}}^{\boldsymbol{s}}(\tau)$ and its connected component $\widehat{\boldsymbol{W}}^{\boldsymbol{s}}(\tau)$, as done in [12]: the case of the unstable set $\widetilde{\boldsymbol{W}}^{\boldsymbol{u}}(\tau)$ and $\widehat{\boldsymbol{W}}^{u}(\tau)$ is analogous. The idea is to entrap the flow of the non-autonomous system (1.6) in a triangular-like set $\boldsymbol{E}^{-}$as done in [12].

We need to introduce some notation that will be in force in the whole Section 3: let us set

$$
\underline{K}(\tau)=\inf \left\{K\left(\mathrm{e}^{t}\right) \mid t \geq \tau\right\}, \quad \bar{K}(\tau)=\sup \left\{K\left(\mathrm{e}^{t}\right) \mid t \geq \tau\right\} .
$$

We denote by $\boldsymbol{E}^{-}(\tau), \boldsymbol{\partial} \boldsymbol{E}^{-}(\tau), \widehat{\ell}(\tau), \boldsymbol{A}(\tau), \boldsymbol{B}(\tau)$ and so on respectively the sets $\boldsymbol{E}^{-}, \boldsymbol{\partial} \boldsymbol{E}^{-}, \widehat{\boldsymbol{\ell}}, \boldsymbol{A}, \boldsymbol{B}$ obtained by setting $a=\underline{K}(\tau)$ and $b=\bar{K}(\tau)$. 
Lemma 3.6. Assume (H2) then there is $\mathcal{T} \in \mathbb{R}$ such that, for any $\tau \geq \mathcal{T}$, the sets $\widetilde{\boldsymbol{W}^{\boldsymbol{s}}}(\tau)$ contain compact and connected continua $\widehat{\boldsymbol{W}}^{\boldsymbol{s}}(\tau)$ which contain the origin and a point in $\widehat{\ell}(\tau)$.

Proof. Since $\lim _{r \rightarrow+\infty} K(r)=K(\infty) \in(0,+\infty)$, we can choose $\mathcal{T} \in \mathbb{R}$ such that

$$
\frac{\bar{K}(\tau)}{\underline{K}(\tau)}<\frac{n}{n-p} \quad \text { for any } \tau \geq \mathcal{T}
$$

i.e. condition (H1) holds for any $r \geq \mathrm{e}^{\mathcal{T}}$. Therefore we can apply Lemma 3.1 and Theorem 3.2 for any $\tau \geq \mathcal{T}$ and we get the existence of $\widetilde{\boldsymbol{W}}^{\boldsymbol{s}}(\tau)$ and of $\widehat{\boldsymbol{W}}^{\boldsymbol{s}}(\tau)$, having the desired properties.

In [12] we have denoted by $\boldsymbol{\xi}^{+}(\tau)=\widetilde{\boldsymbol{W}^{\boldsymbol{u}}}(\tau) \cap \widehat{\boldsymbol{\ell}}(\tau)$ and by $\boldsymbol{\xi}^{-}(\tau)=\widetilde{\boldsymbol{W}}^{\boldsymbol{s}}(\tau) \cap$ $\widehat{\boldsymbol{\ell}}(\tau)$. Here we correct this quite bad notation and we set $\widetilde{\boldsymbol{\xi}}^{\boldsymbol{u}}(\tau)=\widetilde{\boldsymbol{W}}^{\boldsymbol{u}}(\tau) \cap \widehat{\boldsymbol{\ell}}(\tau)$, $\widetilde{\boldsymbol{\xi}^{\boldsymbol{s}}}(\tau)=\widetilde{\boldsymbol{W}}^{\boldsymbol{s}}(\tau) \cap \widehat{\boldsymbol{\ell}}(\tau), \widehat{\boldsymbol{\xi}}^{\boldsymbol{u}}(\tau)=\widehat{\boldsymbol{W}}^{\boldsymbol{u}}(\tau) \cap \widehat{\boldsymbol{\ell}}(\tau)$ and $\widehat{\boldsymbol{\xi}}^{\boldsymbol{s}}(\tau)=\widehat{\boldsymbol{W}}^{\boldsymbol{s}}(\tau) \cap \widehat{\boldsymbol{\ell}}(\tau)$.

Let $\varepsilon>0$ be a small constant to be fixed below. We denote by

$$
\begin{aligned}
\boldsymbol{U}_{\boldsymbol{\varepsilon}}^{+} & :=\left\{\boldsymbol{Q}=\left.\left(Q_{1}, Q_{2}\right)\left|\alpha Q_{1}+Q_{2}\right| Q_{2}\right|^{(2-p) /(p-1)}>\varepsilon\right\}, \\
\boldsymbol{\ell}_{\boldsymbol{\varepsilon}} & :=\left\{\boldsymbol{Q}=\left.\left(Q_{1}, Q_{2}\right)\left|\alpha Q_{1}+Q_{2}\right| Q_{2}\right|^{(2-p) /(p-1)}=\varepsilon\right\} .
\end{aligned}
$$

REMARK 3.7. Let $\mathcal{T}$ be as in Lemma 3.6, then we can find $\varepsilon_{0}$ with the following property. For any $0<\varepsilon \leq \varepsilon_{0}, \tau>\mathcal{T}+1$ and $\boldsymbol{P}^{\boldsymbol{s}}(\tau) \in \widetilde{\boldsymbol{\xi}}^{\boldsymbol{s}}(\tau)$ we can find $T_{\varepsilon} \in(\mathcal{T}, \tau)$, such that $\boldsymbol{x}\left(\tau, \boldsymbol{P}^{\boldsymbol{s}}(\tau) ; t\right) \in \boldsymbol{U}^{-}$when $t \geq \tau, \boldsymbol{x}\left(\tau, \boldsymbol{P}^{\boldsymbol{s}}(\tau) ; t\right) \in \boldsymbol{U}^{+}$ when $t \in\left(T_{\varepsilon}, \tau\right)$ and $\boldsymbol{x}\left(\tau, \boldsymbol{P}^{\boldsymbol{s}}(\tau) ; t\right) \in \boldsymbol{U}_{\boldsymbol{\varepsilon}}^{+}$when $t$ is in a (sufficiently small) left neighbourhood of $T_{\varepsilon}$.

Proof. Since $\dot{x}_{1}\left(\tau, \boldsymbol{P}^{\boldsymbol{s}}(\tau) ; \tau\right)=0$ and $\dot{x}_{2}\left(\tau, \boldsymbol{P}^{\boldsymbol{s}}(\tau) ; \tau\right)<0$, the Remark follows from a standard continuity argument.

We introduce now the segments

$$
\begin{aligned}
\boldsymbol{L}^{\boldsymbol{r}}(\mathcal{T}) & :=\left\{\left(x_{1}, x_{2}\right) \notin \boldsymbol{U}_{\varepsilon}^{+} \mid x_{1}=A_{1}(\mathcal{T}), x_{2}>A_{2}(\mathcal{T})\right\}, \\
\boldsymbol{L}^{l}(\mathcal{T}) & :=\left\{\left(x_{1}, x_{2}\right) \notin \boldsymbol{U}_{\varepsilon}^{+} \mid x_{2}=-\left[x_{1}-B_{1}(\mathcal{T})\right]+B_{2}(\mathcal{T}), x_{1}<B_{1}(\mathcal{T})\right\},
\end{aligned}
$$

and we denote by $\boldsymbol{\Lambda}_{\varepsilon}(\mathcal{T})$ the compact, trapezoidal-like set, enclosed by $\boldsymbol{L}^{\boldsymbol{r}}(\mathcal{T})$, $\widehat{\ell}(\mathcal{T}), \boldsymbol{L}^{l}(\mathcal{T})$ and $\boldsymbol{\ell}_{\varepsilon}$, see Figure 2 .

REMARK 3.8. Possibly choosing a smaller $\varepsilon_{0}>0$, we can assume that the flow of (1.6) on $\boldsymbol{L}^{r}(\mathcal{T})$ and $\boldsymbol{L}^{\boldsymbol{l}}(\mathcal{T})$ points towards the exterior of $\boldsymbol{\Lambda}_{\boldsymbol{\varepsilon}}(\mathcal{T})$ for any $t \geq \mathcal{T}$, whenever $0<\varepsilon \leq \varepsilon_{0}$.

Proof. The claim concerning $\boldsymbol{L}^{\boldsymbol{r}}(\mathcal{T})$ follows observing that $\boldsymbol{\Lambda}_{\boldsymbol{\varepsilon}}(\mathcal{T}) \subset \boldsymbol{U}^{+}$. Further, by construction, there is $m>0$ such that $f_{2}(\boldsymbol{B}(\mathcal{T}), t) \leq-2 m<0$ for any $t \geq \mathcal{T}$; so we can assume without loss of generality that $f_{2}(\boldsymbol{Q}, t)<-m$ for 


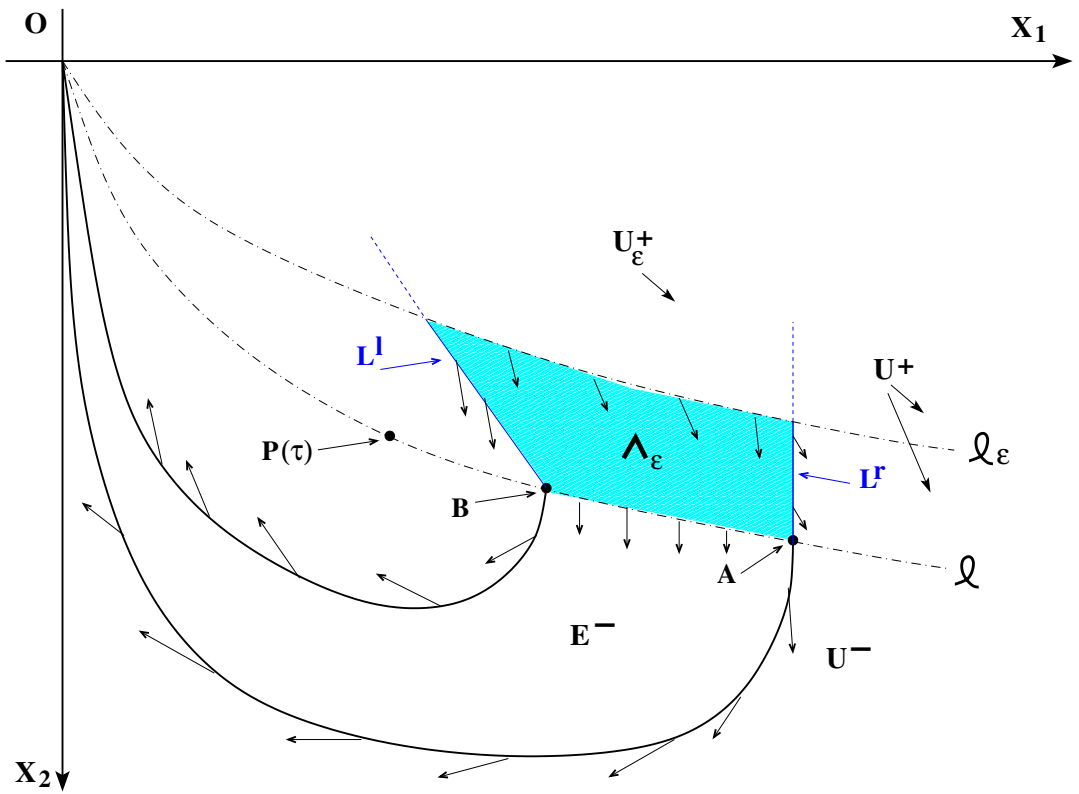

Figure 2. A sketch of the proof of the existence of $\widetilde{\boldsymbol{W}}^{s}(\tau)$ and $\widehat{\boldsymbol{W}}^{\boldsymbol{s}}(\tau)$. When (H1) is assumed we just need to work in $\boldsymbol{U}^{-} \cup \boldsymbol{\ell}$ while when $\boldsymbol{H} \mathbf{2}$ holds we need a truncation argument which involves all the sets defined in the picture.

any $\boldsymbol{Q} \in \boldsymbol{L}^{\boldsymbol{l}}(\mathcal{T})$. Recalling that $0 \leq f_{1}(\boldsymbol{Q}) \leq \varepsilon$ whenever $\boldsymbol{Q} \in \boldsymbol{\Lambda}_{\boldsymbol{\varepsilon}}(\mathcal{T})$ and $t \geq \mathcal{T}$, simply choosing $\varepsilon_{0} \leq m$ we find

$$
f_{2}(\boldsymbol{Q}, t)+f_{1}(\boldsymbol{Q})<-m+\varepsilon \leq 0 .
$$

So the claim concerning $\boldsymbol{L}^{l}(\mathcal{T})$ follows.

Let us set $M=\sup \left\{\left|f_{2}(\boldsymbol{x}, t)\right| \mid \boldsymbol{x} \in \boldsymbol{\Lambda}_{\boldsymbol{\varepsilon}}(\mathcal{T}), t \geq \mathcal{T}\right\}>0$. Now we introduce a modified system which coincides with (1.6) for any $\boldsymbol{Q} \in \boldsymbol{U}^{-}$, but which is constant and $\dot{\boldsymbol{x}} \equiv(0,-M)$ in $\boldsymbol{U}_{\boldsymbol{\varepsilon}}^{+}$. Even if it is not really needed we adjust things in such a way that the modified system is smooth if $x_{2}<0<x_{1}$.

Let $\psi(a): \mathbb{R} \rightarrow[0,+\infty)$ be a $C^{\infty}$ monotone decreasing function such that $\psi(a) \equiv 1$ if $a \leq 0,0<\psi(a)<1$ if $0<a<\varepsilon$, and $\psi(a) \equiv 0$ if $a \geq \varepsilon$. We set

$$
\left\{\begin{array}{l}
\dot{x}_{1}=f_{1}^{\bmod }(\boldsymbol{x}):=f_{1}(\boldsymbol{x}) \psi\left(f_{1}(\boldsymbol{x})\right), \\
\dot{x}_{2}=f_{2}^{\bmod }(\boldsymbol{x} ; t):=f_{2}(x ; t) \psi\left(f_{1}(\boldsymbol{x})\right)-M\left[1-\psi\left(f_{1}(\boldsymbol{x})\right)\right] .
\end{array}\right.
$$

We denote by $\boldsymbol{x}^{\bmod }(\tau, \boldsymbol{Q} ; t)=\left(x_{1}^{\bmod }(\tau, \boldsymbol{Q} ; t), x_{2}^{\bmod }(\tau, \boldsymbol{Q} ; t)\right)$ the trajectory of (3.4) which is in $\boldsymbol{Q}$ at $t=\tau$. Similarly we denote with the apex "mod" all the quantities referred to system (3.4). Our purpose is to reprove Remark 3.7 for system (3.4). We begin by reproving Remark 3.8. 
REMARK 3.9. Let $\varepsilon_{0}$ be as in Remark 3.8; then the flow of (3.4) on $\boldsymbol{L}^{\boldsymbol{r}}(\mathcal{T})$ and $\boldsymbol{L}^{l}(\mathcal{T})$ points towards the exterior of $\boldsymbol{\Lambda}_{\boldsymbol{\varepsilon}}(\mathcal{T})$ for any $t \geq \mathcal{T}$, whenever $0<\varepsilon \leq \varepsilon_{0}$.

Proof. Let $\boldsymbol{Q} \in \boldsymbol{\Lambda}_{\boldsymbol{\varepsilon}}(\mathcal{T})$; recalling that $f_{2}(\boldsymbol{Q} ; t)>-M$ and that $f_{2}^{\bmod }(\boldsymbol{Q} ; t)$ is a convex combination of $f_{2}(\boldsymbol{Q} ; t)$ and $-M$, by construction we have

$$
\begin{aligned}
& 0 \leq f_{1}^{\bmod }(\boldsymbol{Q}) \leq f_{1}(\boldsymbol{Q}), \quad \text { whenever } \boldsymbol{Q} \in \boldsymbol{\Lambda}_{\boldsymbol{\varepsilon}}(\mathcal{T}), \\
& f_{2}^{\bmod }(\boldsymbol{Q} ; t) \leq f_{2}(\boldsymbol{Q} ; t)<0, \quad \text { whenever } \boldsymbol{Q} \in \boldsymbol{\Lambda}_{\boldsymbol{\varepsilon}}(\mathcal{T}) \text { and } t \geq \mathcal{T} .
\end{aligned}
$$

Since $f_{1}^{\bmod }(\boldsymbol{Q})>0$ on $\boldsymbol{L}^{\boldsymbol{r}}(\mathcal{T})$ the claim concerning $\boldsymbol{L}^{\boldsymbol{r}}(\mathcal{T})$ follows. Further from (3.5) we get

$$
f_{2}^{\bmod }(\boldsymbol{Q} ; t)+f_{1}^{\bmod }(\boldsymbol{Q}) \leq f_{2}(\boldsymbol{Q} ; t)+f_{1}(\boldsymbol{Q})
$$

Moreover, the right hand side of (3.6) is negative if $\boldsymbol{Q} \in \boldsymbol{L}^{\boldsymbol{l}}(\mathcal{T})$ and $t \geq \mathcal{T}$, cf. Remark 3.8, whence the claim concerning $\boldsymbol{L}^{\boldsymbol{l}}(\mathcal{T})$ follows and Remark 3.9 is proved.

Lemma 3.10. Let $\mathcal{T}$ be as in Lemma 3.6. For any $0<\varepsilon \leq \varepsilon_{0}, \tau \geq \mathcal{T}+1$, and any $\boldsymbol{P}^{\boldsymbol{s}}(\tau) \in \widetilde{\boldsymbol{\xi}}^{\boldsymbol{s}}(\tau)$ we can find $T_{\varepsilon}^{\bmod } \in(\mathcal{T}, \tau)$ (depending on $\boldsymbol{P}^{\boldsymbol{s}}(\tau)$ ), such that the trajectory $\boldsymbol{x}^{\bmod }\left(\tau, \boldsymbol{P}^{\boldsymbol{s}}(\tau) ; t\right) \in \boldsymbol{U}^{-}$when $t \geq \tau, \boldsymbol{x}^{\bmod }\left(\tau, \boldsymbol{P}^{\boldsymbol{s}}(\tau) ; t\right) \in \boldsymbol{U}^{+}$ when $t<\tau$ and $\boldsymbol{x}^{\bmod }\left(\tau, \boldsymbol{P}^{\boldsymbol{s}}(\tau) ; t\right) \in \boldsymbol{U}_{\boldsymbol{\varepsilon}}^{+}$when $t<T_{\varepsilon}^{\bmod }$.

Proof. From Remark 3.9 we know that either $\boldsymbol{x}\left(\tau, \boldsymbol{P}^{\boldsymbol{s}}(\tau) ; t\right)$ lies in the interior of $\boldsymbol{\Lambda}_{\varepsilon}(\mathcal{T})$ for any $t \in[\mathcal{T}, \tau)$ or there is $T_{\varepsilon}^{\bmod } \in(\mathcal{T}, \tau)$ such that $\boldsymbol{x}\left(\tau, \boldsymbol{P}^{\boldsymbol{s}}(\tau) ; t\right) \in \boldsymbol{\Lambda}_{\boldsymbol{\varepsilon}}(\mathcal{T})$ when $t \in\left[T_{\varepsilon}^{\text {mod }}, \tau\right]$ and it crosses transversally $\boldsymbol{\ell}_{\boldsymbol{\varepsilon}}$ at $t=T_{\varepsilon}^{\bmod }$, and we are done.

Notice that $\dot{x}_{2}^{\bmod }\left(\tau, \boldsymbol{P}^{\boldsymbol{s}}(\tau) ; t\right)$ is uniformly negative when $t \in(\mathcal{T}, \tau)$ and that $\tau-\mathcal{T} \geq 1$, hence the Lemma easily follows by choosing $\varepsilon$ small enough.

Proposition 3.11. Assume (H2), then for any $\tau \in \mathbb{R}$ the sets $\widetilde{\boldsymbol{W}} \boldsymbol{s , \operatorname { m o d }}(\tau)$ contain compact and connected continua $\widehat{\boldsymbol{W}}^{s, \bmod }(\tau)$, which contain the origin and a point in $\boldsymbol{\ell}$.

Proof. When $\tau \geq \mathcal{T}+1$, Proposition 3.11 immediately follows from Lemma 3.6, so let us assume $\tau<\mathcal{T}+1$. Let us consider the connected stable set $\widehat{\boldsymbol{W}}^{\boldsymbol{s}}$, mod $(\mathcal{T}+1)$ which contains the origin and $\boldsymbol{P}^{\boldsymbol{s}}(\tau) \in \widehat{\boldsymbol{\xi}}^{\boldsymbol{s}}(\tau)$, and let us denote by $\widehat{\boldsymbol{M}}^{\boldsymbol{s}, \bmod }(\mathcal{T}+1 ; \tau)$ its image through the flow of $(3.4)$, i.e.

$$
\widehat{\boldsymbol{M}}^{\boldsymbol{s}, \bmod }(\mathcal{T}+1 ; \tau):=\left\{\boldsymbol{x}^{\bmod }(\mathcal{T}+1, \boldsymbol{Q} ; \tau) \mid \boldsymbol{Q} \in \widehat{\boldsymbol{W}}^{\boldsymbol{s}, \bmod }(\mathcal{T}+1)\right\} .
$$

From Lemma 3.10 we know that $\widehat{M}^{s, \bmod }(\mathcal{T}+1 ; \tau) \subset \boldsymbol{U}^{-}$for any $\tau>\mathcal{T}+1$, while if $\tau<\mathcal{T}+1$ it contains $\boldsymbol{X}^{\boldsymbol{s}}(\tau):=\boldsymbol{x}^{\bmod }\left(\mathcal{T}+1, \boldsymbol{P}^{\boldsymbol{s}}(\mathcal{T}+1) ; \tau\right) \in \boldsymbol{U}^{+}$ (in fact $\boldsymbol{X}^{\boldsymbol{s}}(\tau) \in \boldsymbol{U}_{\boldsymbol{\varepsilon}}^{+}$when $\left.\tau<T_{\varepsilon}^{\bmod }\right)$. Since $\widehat{\boldsymbol{M}}^{\boldsymbol{s}}, \bmod (\mathcal{T}+1 ; \tau)$ is connected it follows that $\boldsymbol{\xi}_{\boldsymbol{M}}^{\boldsymbol{s}, \bmod }(\tau):=\left(\widehat{\boldsymbol{M}}^{\boldsymbol{s}, \bmod }(\mathcal{T}+1 ; \tau) \cap \boldsymbol{\ell}\right) \neq \emptyset$ whenever $\tau \leq \mathcal{T}+1$. Then from elementary argument it is easy to check that there is a subset $\widehat{\boldsymbol{W}}^{s, \bmod }(\tau)$ 
of $\widehat{\boldsymbol{M}}^{\boldsymbol{s}, \bmod }(\mathcal{T}+1 ; \tau)$ which is connected, intersects $\boldsymbol{\ell}$ in $\emptyset \neq \boldsymbol{\xi}_{\boldsymbol{M}}^{\boldsymbol{s}, \bmod }(\tau) \subset \widetilde{\boldsymbol{\xi}}^{\boldsymbol{s}, \bmod }(\tau)$ and it is contained in $\widetilde{\boldsymbol{W}}^{\boldsymbol{s}}, \bmod (\tau)$, so Proposition 3.11 is proved.

Proof of Theorem 3.5. Theorem 3.5 now simply follows from Proposition 3.11 by observing that system (1.6) and (3.4) coincide for any $\boldsymbol{x} \in\left(\boldsymbol{U}^{-} \cup \boldsymbol{\ell}\right)$.

\section{From a perturbative to a constructive argument: existence and multiplicity of Ground States}

In this section we give an outline of the proofs of all the main results of [12], explaining what needs a change or a discussion.

The starting point in the whole analysis is the construction of $\widehat{\boldsymbol{W}}^{\boldsymbol{u}}(\tau)$ and $\widehat{\boldsymbol{W}}^{\boldsymbol{s}}(\tau)$, which has been addressed in Section 3. Then the goal is to prove the existence of $\boldsymbol{Q}^{*} \in\left[\widehat{\boldsymbol{\xi}}^{\boldsymbol{u}}\left(\tau^{*}\right) \cap \widehat{\boldsymbol{\xi}}^{\boldsymbol{s}}\left(\tau^{*}\right)\right]$. Whence the existence of GS with FD is immediately obtained through the following result.

LEMma 4.1. Assume that there are $\tau^{*} \in \mathbb{R}$, and $\boldsymbol{Q}^{*} \in\left[\widehat{\boldsymbol{\xi}}^{\boldsymbol{u}}\left(\tau^{*}\right) \cap \widehat{\boldsymbol{\xi}} \boldsymbol{s}\left(\tau^{*}\right)\right] \neq \emptyset$ : then $\boldsymbol{x}\left(\tau^{*}, \boldsymbol{Q}^{*} ; t\right)$ is a homoclinic trajectory and $x_{1}\left(\tau^{*}, \boldsymbol{Q}^{*} ; t\right)>0$ for any $t \in \mathbb{R}$, and the corresponding solution $u(r)$ is a GS with FD.

ProOF. By construction

$$
\begin{array}{ll}
\boldsymbol{x}\left(\tau^{*}, \boldsymbol{Q}^{*} ; t\right) \in \boldsymbol{E}^{+}\left(\tau^{*}\right) & \text { for any } t<\tau^{*}, \\
\boldsymbol{x}\left(\tau^{*}, \boldsymbol{Q}^{*} ; t\right) \in \boldsymbol{E}^{-}\left(\tau^{*}\right) & \text { for any } t>\tau^{*},
\end{array}
$$

whence the Lemma immediately follows.

Assume (H1); let $\boldsymbol{Q}^{\boldsymbol{u}}(\tau), \boldsymbol{Q}^{\boldsymbol{s}}(\tau)$ be points in $\widetilde{\boldsymbol{\xi}}^{\boldsymbol{u}}(\tau)$ and $\widetilde{\boldsymbol{\xi}}^{\boldsymbol{s}}(\tau)$, respectively. To reach our goal, i.e. to prove the existence of $\boldsymbol{Q}^{*} \in\left[\widehat{\boldsymbol{\xi}}^{\boldsymbol{u}}\left(\tau^{*}\right) \cap \widehat{\boldsymbol{\xi}}^{\boldsymbol{s}}\left(\tau^{*}\right)\right]$, we need to give an estimate from above and below of the functions

$$
\boldsymbol{x}^{\boldsymbol{u}}(\tau, t)=\boldsymbol{x}\left(\tau, \boldsymbol{Q}^{\boldsymbol{u}}(\tau) ; t\right) \quad \text { and } \quad \boldsymbol{x}^{\boldsymbol{s}}(\tau, t)=\boldsymbol{x}\left(\tau, \boldsymbol{Q}^{\boldsymbol{s}}(\tau) ; t\right) .
$$

In particular we need to construct functions $\underline{x}_{1}^{u}(\tau ; t), \bar{x}_{1}^{u}(\tau ; t), \underline{x}_{1}^{s}(\tau ; t), \bar{x}_{1}^{s}(\tau ; t)$ with the same asymptotic behavior as $x_{1}^{u}$ and $x_{1}^{s}$ and such that

$$
\begin{aligned}
& \underline{x}_{1}^{u}(t) \leq x_{1}^{u}(\tau ; t+\tau) \leq \bar{x}_{1}^{u}(\tau ; t) \quad \text { for any } t \leq 0, \\
& \underline{x}_{1}^{s}(t) \leq x_{1}^{s}(\tau ; t+\tau) \leq \bar{x}_{1}^{s}(\tau ; t) \quad \text { for any } t \geq 0 \text {, } \\
& \underline{x}_{1}^{u}(t) \sim \mathrm{e}^{\alpha t}, \quad \bar{x}_{1}^{u}(t) \sim \mathrm{e}^{\alpha t} \quad \text { as } t \rightarrow-\infty, \\
& \underline{x}_{1}^{s}(t) \sim \mathrm{e}^{-\alpha t /(p-1)}, \quad \bar{x}_{1}^{s}(t) \sim \mathrm{e}^{-\alpha t /(p-1)} \quad \text { as } t \rightarrow+\infty .
\end{aligned}
$$

In [12] we had simply chosen $\underline{x}_{1}^{u}(t)=U^{b}(t), \bar{x}_{1}^{u}(t)=U^{a}(t), \underline{x}_{1}^{s}(t)=U^{b}(t)$, $\bar{x}_{1}^{s}(t)=U^{a}(t)$, but this choice is wrong. The correct choice for the functions appearing in (4.1) is the object of Section 4.1, see in particular (4.14) and (4.15). 
Then we need to show that there are $\tau^{1} \neq \tau^{2}$ such that

$$
\begin{aligned}
& H\left(\boldsymbol{Q}^{\boldsymbol{s}}\left(\tau^{1}\right), \tau^{1}\right)<0<H\left(\boldsymbol{Q}^{\boldsymbol{u}}\left(\tau^{1}\right), \tau^{1}\right), \\
& H\left(\boldsymbol{Q}^{\boldsymbol{u}}\left(\tau^{2}\right), \tau^{2}\right)<0<H\left(\boldsymbol{Q}^{\boldsymbol{s}}\left(\tau^{2}\right), \tau^{2}\right),
\end{aligned}
$$

whenever $\boldsymbol{Q}^{\boldsymbol{u}}\left(\tau^{i}\right) \in \widetilde{\boldsymbol{\xi}}^{\boldsymbol{u}}\left(\tau^{i}\right)$ and $\boldsymbol{Q}^{\boldsymbol{s}}\left(\tau^{i}\right) \in \widetilde{\boldsymbol{\xi}}^{\boldsymbol{s}}\left(\tau^{i}\right)$ for $i=1,2$. More precisely, we want to prove the following result.

Lemma 4.2. Assume $\left(\alpha^{+}\right)$and $\left(\Omega^{-}\right)$. Then there are $\tau_{1}<\tau_{2}$ such that (4.2) holds. Assume $\left(\alpha^{-}\right)$and $\left(\Omega^{+}\right)$. Then there are $\tau_{1}>\tau_{2}$ such that $(4.2)$ holds.

A sketch of the proof of this lemma is postponed to Section 4.2. In fact, when $(H 1)$ holds it is obtained by combining (2.2) and (4.1), while when (H2) holds we need to follow the argument of [13, Lemma 3.1], [13, Lemma 3.2]. Then we need the following result.

Lemma 4.3. Assume that either (H1) or (H2) are satisfied, and that there are $\tau^{1} \neq \tau^{2}$ such that (4.2) holds. Then, there is $\tau^{*}$ between $\tau^{1}$ and $\tau^{2}$ such that there is $\boldsymbol{Q}^{*} \in\left(\widehat{\boldsymbol{\xi}}^{\boldsymbol{u}}\left(\tau^{*}\right) \cap \widehat{\boldsymbol{\xi}}^{\boldsymbol{s}}\left(\tau^{*}\right)\right)$.

The existence of a GS with FD is then obtained via Lemma 4.1. The proof of Lemma 4.3 is elementary when (H1) is assumed, but it is not trivial if (H2) is assumed, since in this case the set $\widehat{\boldsymbol{\zeta}}^{\boldsymbol{s}}:=\left\{(\boldsymbol{Q}, \tau) \mid \boldsymbol{Q} \in \widehat{\boldsymbol{\xi}}^{\boldsymbol{s}}(\tau)\right\}$ may be disconnected, even in the smooth case $p=2$ where $\widehat{\boldsymbol{W}}^{\boldsymbol{s}}(\tau)$ is indeed a manifold. The proof of this lemma, which is not present in [12], is postponed to Section 4.2.

To complete the proof of Theorems 1.3 and 1.4 of this addendum, and all the results of $[12, \S 2]$, now we simply have to put together Lemmas $4.2,4.3$ and 4.1 to get the existence of at least one GS with FD, and to argue as in [13, Theorem 3.3] to get the existence of the other types of solutions (GS with SD, SGS with FD, SGS with SD, oscillatory solutions).

Further, when (H1) holds, we are in the position to give explicit non-perturbative hypotheses which allow to establish the existence of sequences of values for which $H\left(\boldsymbol{Q}^{\boldsymbol{s}}\left(\tau^{i}\right), \tau^{i}\right)-H\left(\boldsymbol{Q}^{\boldsymbol{u}}\left(\tau^{i}\right), \tau^{i}\right)$ changes sign. Then, applying again Lemmas 4.1 and 4.3 we reprove all the results in $[12, \S 4]$ and we get the existence of many GS with FD, without asking for perturbative conditions.

These hypotheses are expressed through the sign of the following auxiliary functions which can be explicitly computed:

$$
\begin{aligned}
& F_{-}^{u}(\tau):=\int_{-\infty}^{0} \dot{\phi}^{+}(s+\tau) \frac{\left|\underline{x}_{1}^{u}(s)\right|^{\sigma}}{\sigma} d s-\int_{-\infty}^{0} \dot{\phi}^{-}(s+\tau) \frac{\left|\bar{x}_{1}^{u}(s)\right|^{\sigma}}{\sigma} d s, \\
& F_{+}^{u}(\tau):=\int_{-\infty}^{0} \dot{\phi}^{+}(s+\tau) \frac{\left|\bar{x}_{1}^{u}(s)\right|^{\sigma}}{\sigma} d s-\int_{-\infty}^{0} \dot{\phi}^{-}(s+\tau) \frac{\left|\underline{x}_{1}^{u}(s)\right|^{\sigma}}{\sigma} d s \\
& F_{-}^{s}(\tau):=\int_{0}^{\infty} \dot{\phi}^{-}(s+\tau) \frac{\left|\underline{x}_{1}^{s}(s)\right|^{\sigma}}{\sigma} d s-\int_{0}^{\infty} \dot{\phi}^{+}(s+\tau) \frac{\left|\bar{x}_{1}^{s}(s)\right|^{\sigma}}{\sigma} d s
\end{aligned}
$$




$$
F_{+}^{s}(\tau):=\int_{0}^{\infty} \dot{\phi}^{-}(s+\tau) \frac{\left|\bar{x}_{1}^{s}(s)\right|^{\sigma}}{\sigma} d s-\int_{0}^{\infty} \dot{\phi}^{+}(s+\tau) \frac{\left|\underline{x}_{1}^{s}(s)\right|^{\sigma}}{\sigma} d s .
$$

Now, combining (4.1) and (4.28), we can deduce the following:

$$
\begin{array}{ll}
F_{-}^{u}(\tau) \leq H(\boldsymbol{Q}, \tau) \leq F_{+}^{u}(\tau) & \text { for any } \boldsymbol{Q} \in \widetilde{\boldsymbol{\xi}}^{u}(\tau), \\
F_{-}^{s}(\tau) \leq H(\boldsymbol{R}, \tau) \leq F_{+}^{s}(\tau) & \text { for any } \boldsymbol{R} \in \widetilde{\boldsymbol{\xi}}^{s}(\tau) .
\end{array}
$$

Thus, combining Lemmas 4.1 and 4.3, we get the following multiplicity result.

Theorem 4.4. Assume (H1). Assume further that there are $\tau_{1}$ and $\tau_{2}$ such that $F_{-}^{s}\left(\tau_{1}\right) \geq F_{+}^{u}\left(\tau_{1}\right)$ and $F_{-}^{u}\left(\tau_{2}\right) \geq F_{+}^{s}\left(\tau_{2}\right)$. Then there exists at least one homoclinic trajectory of system (1.6), which corresponds to a GS with fast decay of (1.3). Analogously, if we find a sequence $\tau_{0}<\tau_{1}<\ldots<\tau_{k}$, such that

$$
F_{+}^{u}\left(\tau_{2 i+1}\right)-F_{-}^{s}\left(\tau_{2 i+1}\right) \leq 0 \leq F_{+}^{s}\left(\tau_{2 i}\right)-F_{-}^{u}\left(\tau_{2 i}\right)
$$

for any $i$, then (1.3) admits at least $k$ GS with FD. If the sequence $\tau_{k}$ is defined for any $k \in \mathbb{N}$ (e.g. if $\phi(t)$ is periodic or almost periodic) then (1.3) admits infinitely many GS with FD.

4.1. Construction of the auxiliary functions. In this section we always assume $\boldsymbol{H} 1$ and we construct the functions $\underline{x}_{1}^{u}, \bar{x}_{1}^{u}, \underline{x}_{1}^{s}, \bar{x}_{1}^{s}$ appearing in (4.1). The argument is based on some non-trivial ideas developed on the phase portrait, and we need to introduce several sets: we collect here all these definitions and we invite the reader to follow them on Figure 1.

We recall that $\boldsymbol{B}=\left(B_{1}, B_{2}\right)$ and $\boldsymbol{A}=\left(A_{1}, A_{2}\right)$ are the intersections of the isocline $\boldsymbol{\ell}$ respectively with $\boldsymbol{\Gamma}^{a}$ and $\boldsymbol{\Gamma}^{b}$, and that $\boldsymbol{P}(\mathcal{K})$ is the critical point of the frozen system (1.6) where $\phi(t) \equiv \mathcal{K}$, see (2.4). Let $\boldsymbol{Q} \in \boldsymbol{\Gamma}^{a}$ and $\tau \in \mathbb{R}$, we denote by $\boldsymbol{\psi}^{\boldsymbol{a}}(\tau, \boldsymbol{Q} ; t)=\left(\psi_{1}^{a}(\tau, \boldsymbol{Q} ; t), \psi_{2}^{a}(\tau, \boldsymbol{Q} ; t)\right)$ the homoclinic trajectory of the autonomous system (1.6) where $\phi(t) \equiv a$ which is in $\boldsymbol{Q}$ at $t=\tau$. Notice that there is $T=T(\boldsymbol{Q})$ such that $\psi_{1}^{a}(\tau, \boldsymbol{Q} ; t)=U^{a}(t-T)$ and if $\boldsymbol{Q} \in \boldsymbol{\ell}$ then $T=\tau$, i.e.

$$
\psi_{1}^{a}(\tau, \boldsymbol{Q} ; t)=U^{a}(t-\tau)
$$

where $U^{a}(t)$ is given by (2.3). Similarly if $\boldsymbol{Q} \in \boldsymbol{\Gamma}^{\boldsymbol{b}}$ we denote by $\boldsymbol{\psi}^{\boldsymbol{b}}(\tau, \boldsymbol{Q} ; t)$ the homoclinic trajectory of the autonomous system (1.6) where $\phi(t) \equiv b$.

We denote by $\boldsymbol{A}^{+}=\left(B_{1}, A_{2}^{+}\right), \boldsymbol{A}^{-}=\left(B_{1}, A_{2}^{-}\right)$the intersection of the line $x_{1}=B_{1}$ respectively with the curves $\boldsymbol{\Gamma}^{\boldsymbol{a}} \cap \boldsymbol{U}^{+}$and $\boldsymbol{\Gamma}^{\boldsymbol{a}} \cap \boldsymbol{U}^{-}$, so that $A_{2}^{-}<$ $B_{2}<A_{2}^{+}$. Notice that $A_{2}^{+}$and $A_{2}^{-}$are the largest and the smallest (negative) solutions of the following equation in $Y$

$$
\frac{p-1}{p}|Y|^{p /(p-1)}+\left(\frac{\sigma \alpha^{\sigma}}{p b}\right)^{1 /(\sigma-p)} Y+\frac{a}{\sigma}\left(\frac{\sigma \alpha^{p}}{p b}\right)^{\sigma /(\sigma-p)}=0 .
$$


Then we set

$$
\begin{array}{lll}
\overline{\boldsymbol{L}}^{+}=\left\{\left(B_{1}, x_{2}\right) \mid B_{2}<x_{2}<A_{2}^{+}\right\}, & \overline{\boldsymbol{L}}^{-}=\left\{\left(B_{1}, x_{2}\right) \mid A_{2}^{-}<x_{2}<B_{2}\right\}, \\
\overline{\boldsymbol{E}}^{+}=\left\{\boldsymbol{x} \in \boldsymbol{E}^{+} \mid x_{1}<B_{1}\right\}, & \widetilde{\boldsymbol{E}}^{+}=\left\{\boldsymbol{x} \in \boldsymbol{E}^{+} \mid x_{1}>B_{1}\right\}, \\
\overline{\boldsymbol{E}}^{-}=\left\{\boldsymbol{x} \in \boldsymbol{E}^{-} \mid x_{1}<B_{1}\right\}, & \widetilde{\boldsymbol{E}}^{-}=\left\{\boldsymbol{x} \in \boldsymbol{E}^{-} \mid x_{1}>B_{1}\right\} .
\end{array}
$$

Notice that $\boldsymbol{E}^{+}=\overline{\boldsymbol{E}}^{+} \cup \boldsymbol{L}^{+} \cup \widetilde{\boldsymbol{E}}^{+}$and $\boldsymbol{E}^{-}=\overline{\boldsymbol{E}}^{-} \cup \boldsymbol{L}^{-} \cup \widetilde{\boldsymbol{E}}^{-}$. Then we denote by $\boldsymbol{\partial} \overline{\boldsymbol{E}}^{ \pm}=\left(\boldsymbol{\partial E} \cap \overline{\boldsymbol{E}}^{ \pm}\right)$, and by $\boldsymbol{\partial} \widetilde{\boldsymbol{E}}^{ \pm}=\left(\boldsymbol{\partial E} \cap \widetilde{\boldsymbol{E}}^{ \pm}\right)$.

Remark 4.5. Let $\boldsymbol{Q}=\left(Q_{1}, Q_{2}\right) \in \overline{\boldsymbol{E}}^{+}$and $\boldsymbol{Q}^{\boldsymbol{a}}=\left(Q_{1}, Q_{2}^{a}\right) \in\left(\boldsymbol{\Gamma}^{\boldsymbol{a}} \cap \overline{\boldsymbol{E}}^{+}\right)$, $\boldsymbol{Q}^{b}=\left(Q_{1}, Q_{2}^{b}\right) \in\left(\boldsymbol{\Gamma}^{b} \cap \overline{\boldsymbol{E}}^{+}\right)$so that $Q_{2}^{b}<Q_{2}<Q_{2}^{a}<0$. Then

$$
\psi_{1}^{a}\left(\tau, \boldsymbol{Q}^{a} ; t\right)<x_{1}(\tau, \boldsymbol{Q} ; t)<\psi_{1}^{b}\left(\tau, \boldsymbol{Q}^{\boldsymbol{b}} ; t\right), \quad \text { for any } t<\tau .
$$

Analogously, let $\boldsymbol{Q}=\left(Q_{1}, Q_{2}\right) \in \overline{\boldsymbol{E}}^{-}$and $\boldsymbol{Q}^{\boldsymbol{a}}=\left(Q_{1}, Q_{2}^{a}\right) \in\left(\boldsymbol{\Gamma}^{a} \cap \overline{\boldsymbol{E}}^{-}\right), \boldsymbol{Q}^{\boldsymbol{b}}=$ $\left(Q_{1}, Q_{2}^{b}\right) \in\left(\Gamma^{b} \cap \bar{E}^{-}\right)$so that $Q_{2}^{a}<Q_{2}<Q_{2}^{b}<0$. Then

$$
\psi_{1}^{a}\left(\tau, \boldsymbol{Q}^{a} ; t\right)<x_{1}(\tau, \boldsymbol{Q} ; t)<\psi_{1}^{b}\left(\tau, \boldsymbol{Q}^{\boldsymbol{b}} ; t\right), \quad \text { for any } t>\tau .
$$

Proof. The argument of this proof is inspired by [13, p. 358] but it is repeated here for convenience of the reader. We just prove (4.11) since (4.10) is analogous. Notice that in (4.11) we have the equality for $t=\tau$, and by construction

$$
\dot{\psi}_{1}^{a}\left(\tau, \boldsymbol{Q}^{a} ; t\right)<\dot{x}_{1}(\tau, \boldsymbol{Q} ; t)<\dot{\psi}_{1}^{b}\left(\tau, \boldsymbol{Q}^{b} ; t\right)
$$

at $t=\tau$ since $Q_{2}^{a}<Q_{2}<Q_{2}^{b}$. Hence it follows that (4.11) holds in a right neighbourhood of $t=\tau$. Assume by contradiction that there is $T>\tau$ such that (4.11) holds for $t \in(\tau, T)$ and $\psi_{1}^{a}\left(\tau, \boldsymbol{Q}^{a} ; T\right)=x_{1}(\tau, \boldsymbol{Q} ; T)=\psi_{1}^{b}\left(\tau, \boldsymbol{Q}^{b} ; T\right)$; then, again by construction, we see that $\psi_{2}^{a}\left(\tau, \boldsymbol{Q}^{\boldsymbol{a}} ; T\right)<x_{2}(\tau, \boldsymbol{Q} ; T)<\psi_{2}^{b}\left(\tau, \boldsymbol{Q}^{\boldsymbol{b}} ; T\right)$, hence (4.12) holds for $t=T$ too and this gives a contradiction, and proves the claim.

We emphasize that to get the estimate (4.1) we cannot simply use $U^{b}(t-\tau)$ and $U^{a}(t-\tau)$ as we did in [12]. The problem originates from equation (2.10) in [12] which is wrong and should be replaced by a more articulated argument. In fact, we need two different ideas, one for $\overline{\boldsymbol{E}}^{+}$and one for $\widetilde{\boldsymbol{E}}^{+}$(and similarly one for $\overline{\boldsymbol{E}}^{-}$and one for $\widetilde{\boldsymbol{E}}^{-}$). In fact, in $\overline{\boldsymbol{E}}^{+}$we use the argument of [13], which is in fact similar to the one used in [12], i.e. we perform the estimate via Remark 4.5 and equations (4.10) and (4.11). But in $\widetilde{\boldsymbol{E}}^{+}$we need to use a new idea.

More precisely, fix $\tau \in \mathbb{R}$ and let $\boldsymbol{Q}^{\boldsymbol{u}}(\tau)$ be a point in $\widetilde{\boldsymbol{\xi}}^{\boldsymbol{u}}(\tau)$ and $\boldsymbol{Q}^{\boldsymbol{s}}(\tau)$ be a point in $\widetilde{\boldsymbol{\xi}}^{\boldsymbol{s}}(\tau)$. We denote by $T^{u}\left(\boldsymbol{Q}^{\boldsymbol{u}}(\tau)\right)>0$ the value such that $x^{u}(\tau, t) \in$ $\widetilde{\boldsymbol{E}}^{+}$for any $\tau-T^{u}\left(\boldsymbol{Q}^{\boldsymbol{u}}(\tau)\right)<t<\tau$ and it crosses transversally $\overline{\boldsymbol{L}}^{+}$at $t=$ $\tau-T^{u}\left(\boldsymbol{Q}^{u}(\tau)\right)$. Similarly we denote by $T^{s}\left(\boldsymbol{Q}^{s}(\tau)\right)>0$ the value such that $x^{s}(\tau, t) \in \widetilde{\boldsymbol{E}}^{-}$for any $\tau<t<\tau+T^{s}\left(\boldsymbol{Q}^{s}(\tau)\right)$ and it crosses transversally $\overline{\boldsymbol{L}}^{+}$at $t=\tau+T^{s}\left(\boldsymbol{Q}^{s}(\tau)\right)$. 
Lemma 4.6. Assume (H1). Then there are $\widetilde{\mathcal{T}}^{u}>0, \widetilde{\mathcal{T}}^{s}>0$ (explicitly computable), independent of $\tau$, such that $\widetilde{\mathcal{T}}^{u} \geq T^{u}\left(\boldsymbol{Q}^{u}(\tau)\right)$ and $\widetilde{\mathcal{T}}^{s} \geq T^{s}\left(\boldsymbol{Q}^{s}(\tau)\right)$ for any $\tau \in \mathbb{R}, Q^{\boldsymbol{u}}(\tau) \in \widetilde{\boldsymbol{\xi}}^{\boldsymbol{u}}(\tau), \boldsymbol{Q}^{\boldsymbol{s}}(\tau) \in \widetilde{\boldsymbol{\xi}}^{\boldsymbol{s}}(\tau)$.

The proof of this lemma is postponed to Section 4.1.2 (see in particular $(4.25))$.

Further, we denote by $\bar{T}^{u}<0<\bar{T}^{s}$ the values such that

$$
\left(U^{a}\left(\bar{T}^{u}\right), V^{a}\left(\bar{T}^{u}\right)\right)=\boldsymbol{A}^{+}, \quad\left(U^{a}\left(\bar{T}^{s}\right), V^{a}\left(\bar{T}^{s}\right)\right)=\boldsymbol{A}^{-}
$$

We evaluate $\bar{T}^{u}$ and $\bar{T}^{s}$ explicitly in Section 4.1.2, cf. (4.26) and (4.27).

Now we are ready to construct the auxiliary functions $\underline{x}_{1}^{u, s}(t), \bar{x}_{1}^{u, s}(t)$ for which (4.1) holds:

$$
\begin{aligned}
& \bar{x}_{1}^{u}(t):= \begin{cases}A_{1} & \text { if }-\widetilde{\mathcal{T}}^{u}<t \leq 0, \\
U^{b}\left(t+\widetilde{\mathcal{T}}^{u}\right) & \text { if } t \leq-\widetilde{\mathcal{T}}^{u},\end{cases} \\
& \underline{x}_{1}^{u}(t):=U^{a}\left(t+\bar{T}^{u}\right) \quad \text { if } t \leq 0 .
\end{aligned}
$$

Analogously, we define

$$
\begin{aligned}
& \bar{x}_{1}^{s}(t):= \begin{cases}A_{1} & \text { if } 0 \leq t<\widetilde{\mathcal{T}}^{s}, \\
U^{b}\left(t-\widetilde{\mathcal{T}}^{s}\right) & \text { if } t \geq \widetilde{\mathcal{T}}^{s},\end{cases} \\
& \underline{x}_{1}^{u}(t):=U^{a}\left(t+\bar{T}^{s}\right) \quad \text { if } t \geq 0 .
\end{aligned}
$$

The validity of (4.1) then follows from Remark 4.5.

4.1.1. Estimate of $\widetilde{\mathcal{T}}^{u}$ and $\widetilde{\mathcal{T}}^{s}$. In this subsection we always assume (H1). Let $\boldsymbol{Q}^{\boldsymbol{u}}(\tau), \boldsymbol{Q}^{\boldsymbol{s}}(\tau)$ be points respectively in $\widehat{\boldsymbol{\xi}}^{\boldsymbol{u}}(\tau)$ and in $\widehat{\boldsymbol{\xi}}^{\boldsymbol{s}}(\tau)$. We evaluate explicitly the lower bound (uniform in $\tau) \widetilde{\mathcal{T}}^{u}$ for the time $T^{u}\left(\boldsymbol{Q}^{u}(\tau)\right.$ ) spent by the trajectory $\boldsymbol{x}\left(\tau, \boldsymbol{Q}^{\boldsymbol{u}}(\tau) ; t\right)$ in the set $\widetilde{\boldsymbol{E}}^{+}$and the lower bound $\widetilde{\mathcal{T}}^{s}$ for the time $T^{s}\left(\boldsymbol{Q}^{s}(\tau)\right)$ spent by $\boldsymbol{x}\left(\tau, \boldsymbol{Q}^{s}(\tau) ; t\right)$ in the set $\widetilde{\boldsymbol{E}}^{-}$. For this purpose we need to compare the solutions of the original problem (1.6) lying in $\widetilde{\boldsymbol{E}}^{ \pm}$with some auxiliary ones.

First of all we rewrite (1.6) by translating the variables so that $\boldsymbol{P}(a)$ becomes the origin of the new system. Hence we set $\boldsymbol{y}=\left(y_{1}, y_{2}\right)$ where $y_{1}=x_{1}-P_{1}(a)$ and $y_{2}=x_{2}-P_{2}(a)$,

$$
\begin{aligned}
g_{1}\left(y_{2}\right) & :=\left(y_{2}+P_{2}(a)\right)\left|y_{2}+P_{2}(a)\right|^{(2-p) /(p-1)}-P_{2}(a)\left|P_{2}(a)\right|^{(2-p) /(p-1)}, \\
g_{2}\left(y_{1} ; t\right) & :=\phi(t)\left|y_{1}+P_{1}(a)\right|^{\sigma-1}-a P_{1}(a)^{\sigma-1} .
\end{aligned}
$$

Using the fact that $\boldsymbol{P}(a)$ is a critical point of the frozen system where $K \equiv a$, we get:

$$
\left\{\begin{array}{l}
\dot{y}_{1}=\alpha y_{1}+g_{1}\left(y_{2}\right), \\
\dot{y}_{2}=-\alpha y_{2}-g_{2}\left(y_{1} ; t\right) .
\end{array}\right.
$$


We denote by

$$
\begin{aligned}
& \widetilde{\boldsymbol{E}}_{\boldsymbol{y}}^{ \pm}:=\left\{\boldsymbol{y}=\boldsymbol{x}-\boldsymbol{P}(a) \mid \boldsymbol{x} \in \widetilde{\boldsymbol{E}}^{ \pm}\right\}, \\
& \boldsymbol{\partial} \widetilde{\boldsymbol{E}}_{\boldsymbol{y}}^{ \pm}:=\left\{\boldsymbol{y}=\boldsymbol{x}-\boldsymbol{P}(a) \mid \boldsymbol{x} \in \boldsymbol{\partial} \widetilde{\boldsymbol{E}}^{ \pm}\right\} .
\end{aligned}
$$

From Lemma 2.2 it follows that the flow of (4.17) on $\boldsymbol{\partial} \widetilde{\boldsymbol{E}}_{\boldsymbol{y}}^{+}$points towards the interior of $\widetilde{\boldsymbol{E}}_{\boldsymbol{y}}^{+}$while on $\boldsymbol{\partial} \widetilde{\boldsymbol{E}}_{\boldsymbol{y}}^{-}$it points towards the exterior of $\widetilde{\boldsymbol{E}}_{\boldsymbol{y}}^{-}$. Further $\boldsymbol{P}(a) \notin\left(\widetilde{\boldsymbol{E}}^{+} \cup \widetilde{\boldsymbol{E}}^{-}\right)$, hence $(0,0) \notin\left(\widetilde{\boldsymbol{E}}_{\boldsymbol{y}}^{+} \cup \widetilde{\boldsymbol{E}}_{\boldsymbol{y}}^{-}\right)$and for any trajectory starting from $\widetilde{\boldsymbol{E}}_{\boldsymbol{y}}^{ \pm}$we can define the angular coordinates $\boldsymbol{y}(t)=R(t)(\cos (\theta(t)), \sin (\theta(t)))$ and we get

$$
-\dot{\theta}=\frac{2 \alpha y_{1} y_{2}+y_{1} g_{2}\left(y_{1} ; t\right)+y_{2} g_{1}\left(y_{2}\right)}{R^{2}} .
$$

We want to compare (4.17) with autonomous linear systems having angular speed negative but larger than (4.17), more precisely with the following

$$
\left\{\begin{array}{l}
\dot{y}_{1}=\alpha y_{1}+g_{1}^{*} y_{2}, \\
\dot{y}_{2}=-\alpha y_{2}-g_{2}^{*} y_{1},
\end{array}\right.
$$

where $g_{1}^{*}$ and $g_{2}^{*}$ are positive constants which will be determined just below.

We stress that there are $\chi^{+} \leq \chi^{-}<0$ such that

$$
\begin{aligned}
& \widetilde{\boldsymbol{E}}_{\boldsymbol{y}}^{+} \subset\left\{\boldsymbol{y} \mid y_{2} \geq \chi^{+} y_{1}, y_{1} \geq 0\right\}, \\
& \widetilde{\boldsymbol{E}}_{\boldsymbol{y}}^{-} \subset\left\{\boldsymbol{y} \mid y_{2} \leq \chi^{-} y_{1}, y_{1} \geq 0\right\},
\end{aligned}
$$

where

$$
\begin{aligned}
\text { if } p=2 & \Rightarrow \chi^{+}=\chi^{-}=-\frac{1}{\alpha}, \\
\text { if } p>2 & \Rightarrow \chi^{+}=\frac{A_{2}-B_{2}}{A_{1}-B_{1}}, \quad \chi^{-}=-(p-1)\left[\alpha B_{1}\right]^{p-2}, \\
\text { if } 1<p<2 & \Rightarrow \chi^{+}=-(p-1)\left[\alpha B_{1}\right]^{p-2}, \quad \chi^{-}=\frac{A_{2}-B_{2}}{A_{1}-B_{1}} .
\end{aligned}
$$

We observe that if $\boldsymbol{y}=\left(y_{1}, y_{2}\right) \in\left(\boldsymbol{E}_{\boldsymbol{y}}^{+} \cup \boldsymbol{E}_{\boldsymbol{y}}^{-}\right)$then $P_{1}(a)<B_{1}<y_{1}+P_{1}(a)<A_{1}$. Further, if $\sigma \geq 2$ (in particular if $p \geq 2$ ), then $g_{2}$ is convex for $y_{1}>0$, hence

$$
g_{2}\left(y_{1} ; t\right) \geq a\left[\left|y_{1}+P_{1}(a)\right|^{\sigma-1}-P_{1}(a)^{\sigma-1}\right] \geq g_{2}^{*} y_{1}
$$

where

$$
g_{2}^{*}:=a(\sigma-1) P_{1}(a)^{\sigma-2} .
$$

Similarly, if $1<p<\sigma<2$, then $g_{2}$ is concave for $y_{1}>0$; hence, if $\boldsymbol{y}=$ $\left(y_{1}, y_{2}\right) \in\left(\boldsymbol{E}_{\boldsymbol{y}}^{+} \cup \boldsymbol{E}_{\boldsymbol{y}}^{-}\right)$, then

$$
g_{2}\left(y_{1} ; t\right) \geq a\left[\left|y_{1}+P_{1}(a)\right|^{\sigma-1}-P_{1}(a)^{\sigma-1}\right] \geq g_{2}^{*} y_{1}
$$

where

$$
g_{2}^{*}:=a \frac{A_{1}^{\sigma-1}-P_{1}(a)^{\sigma-1}}{A_{1}-P_{1}(a)}>a(\sigma-1) A_{1}^{\sigma-2} .
$$


To estimate $g_{1}$ we need to distinguish the cases $p=2, p>2$ and $1<p<2$. First observe that, if $\boldsymbol{y}=\left(y_{1}, y_{2}\right) \in \boldsymbol{E}_{\boldsymbol{y}}^{+}$, then $A_{2}<y_{2}+P_{2}(a)<A_{2}^{+}$, while if $\boldsymbol{y}=\left(y_{1}, y_{2}\right) \in \boldsymbol{E}_{\boldsymbol{y}}^{-}$then $A_{2}^{-}<y_{2}+P_{2}(a)<P_{2}(a)$.

- If $p=2$ we simply have $g_{1}\left(y_{2}\right)=g_{1}^{*} y_{2}$ where $g_{1}^{*}:=1$.

- If $p>2$ then $g_{1}$ is convex whenever $\boldsymbol{y} \in\left(\boldsymbol{E}_{\boldsymbol{y}}^{+} \cup \boldsymbol{E}_{\boldsymbol{y}}^{-}\right)$(since $y_{2}+P_{2}(a)<$ $0)$, therefore

$$
g_{1}\left(y_{2}\right) \geq g_{1}^{*} y_{2} \quad \text { where } g_{1}^{*}:=\frac{\left|P_{2}(a)\right|^{(2-p) /(p-1)}}{p-1} .
$$

- If $1<p<2$ then $g_{1}$ is concave whenever $\boldsymbol{y} \in\left(\boldsymbol{E}_{\boldsymbol{y}}^{+} \cup \boldsymbol{E}_{\boldsymbol{y}}^{-}\right)$, hence

$$
g_{1}\left(y_{2}\right) \geq g_{1}^{*}\left(y_{2}\right) y_{2} \quad \text { where } g_{1}^{*}\left(y_{2}\right):= \begin{cases}\frac{g_{1}\left(A_{2}^{+}-P_{2}(a)\right)}{A_{2}^{+}-P_{2}(a)} & \text { if } y_{2} \geq 0, \\ \frac{g_{1}\left(A_{2}^{-}-P_{2}(a)\right)}{A_{2}-P_{2}(a)} & \text { if } y_{2} \leq 0 .\end{cases}
$$

Notice that by construction the angular speed of (4.17) is negative and larger in absolute value than the angular speed of the linear system (4.19). Further $g_{1}^{*} g_{2}^{*}-\alpha^{2}>0$ if (H1) holds true, so if we set

$$
\omega=\sqrt{g_{1}^{*} g_{2}^{*}-\alpha^{2}}
$$

from a straightforward computation we see that

$$
\left(y_{1}(t), y_{2}(t)\right)=\left(\cos (\omega t),-\frac{1}{g_{1}^{*}}(\omega \sin (\omega t)+\alpha \cos (\omega t))\right.
$$

is the solution of $(4.19)$ such that $\left(y_{1}(0), \dot{y}_{1}(0)\right)=(1,0)$. Then it is easy to check that any solution of (4.19) lies in $\widetilde{\boldsymbol{E}}_{\boldsymbol{y}}^{+}$for a time smaller or equal to $\widetilde{\mathcal{T}}^{u}$ and stays in $\widetilde{\boldsymbol{E}}_{\boldsymbol{y}}^{-}$for a time smaller or equal to $\widetilde{\mathcal{T}}^{s}$, where

$$
\begin{aligned}
\widetilde{\mathcal{T}}^{u} & =\frac{1}{\omega^{+}}\left[\frac{\pi}{2}+\theta^{+}\right], & \widetilde{\mathcal{T}}^{s} & =\frac{1}{\omega^{-}}\left[\frac{\pi}{2}-\theta^{-}\right], \\
\theta^{+} & =\arctan \left(\frac{\left|\chi^{+}\right| g_{1}^{*}-\alpha}{\omega^{+}}\right), & \theta^{-} & =\arctan \left(\frac{\left|\chi^{-}\right| g_{1}^{*}-\alpha}{\omega^{-}}\right) .
\end{aligned}
$$

4.1.2. Estimate of $\bar{T}^{u}$ and $\bar{T}^{s}$. In this subsection we assume either (H1) or (H2) so that $\widehat{\boldsymbol{W}}^{\boldsymbol{u}}(\tau)$ and $\widehat{\boldsymbol{W}}^{s}(\tau)$ exist. To evaluate $\bar{T}^{u}$ and $\bar{T}^{s}$ we simply proceed using the definition, given in (4.13). Hence $\bar{T}^{u}<0<\bar{T}^{s}$ are respectively the unique negative and positive solutions of the equation in $t$

$$
f(t)=C\left(\frac{b}{a}\right)^{1 / p}
$$


where

$$
\begin{aligned}
f(t) & =\mathrm{e}^{-t(p+\delta) / p}+(p-1) \mathrm{e}^{t(p+\delta) /(p(p-1))}, \\
C & =\frac{p^{1 / p}(p-1)^{(p-1) /(p-2)}}{\alpha \sigma^{1 / p}}(n+\delta)^{1 / p}\left(\frac{n-p}{p-1}\right)^{(p-1) / p} .
\end{aligned}
$$

Notice that $C=2^{3 / 2}$ for $p=2$, and that $f(t)$ is decreasing for $t<0$ and increasing for $t>0$. In fact we can approximate $\bar{T}^{u}<0<\bar{T}^{s}$ as follows

$$
\bar{T}^{u}=-\frac{p}{p+\delta} \ln \left(\frac{C b^{1 / p}}{a^{1 / p}}\right), \quad \bar{T}^{s}=\frac{p(p-1)}{p+\delta} \ln \left(\frac{C b^{1 / p}}{(p-1) a^{1 / p}}\right)
$$

(notice that $\bar{T}^{u}=-\bar{T}^{s}$ if $p=2$ ).

REmark 4.7. We emphasize that (4.27) holds also if assumption (H2) replaces (H1).

4.2. Proof of Lemmas 4.2 and 4.3. Let us begin by giving a sketch of the proof of Lemma 4.2.

Proof of Lemma 4.2. Assume first that $\boldsymbol{H} \mathbf{1}$ holds so that we can construct the functions $\underline{x}_{1}^{u, s}, \bar{x}_{1}^{u, s}$ as done in Section 4.1, and (4.1) holds. Following [12] we set $\dot{\phi}^{+}(t)=\max \{\dot{\phi}(t), 0\}$ and $\dot{\phi}^{-}(t)=\max \{-\dot{\phi}(t), 0\}$. Observe that if $\boldsymbol{Q}^{\boldsymbol{u}} \in$ $\widehat{\boldsymbol{\xi}}^{\boldsymbol{u}}(\tau)$ and $\boldsymbol{Q}^{s} \in \widehat{\boldsymbol{\xi}}^{s}(\tau)$ then

$$
\begin{aligned}
H\left(\boldsymbol{Q}^{u}, \tau\right) & =\int_{-\infty}^{\tau} \dot{\phi}(s) \frac{\left|x_{1}^{u}(\tau ; s)\right|^{\sigma}}{\sigma} d s \\
& =\int_{-\infty}^{0} \dot{\phi}(t+\tau) \frac{\left|x_{1}^{u}(\tau ; t+\tau)\right|^{\sigma}}{\sigma} d t, \\
H\left(\boldsymbol{Q}^{s}, \tau\right) & =-\int_{\tau}^{+\infty} \dot{\phi}(s) \frac{\left|x_{1}^{s}(\tau ; s)\right|^{\sigma}}{\sigma} d s \\
& =-\int_{0}^{+\infty} \dot{\phi}(t+\tau) \frac{\left|x_{1}^{s}(\tau ; t+\tau)\right|^{\sigma}}{\sigma} d t .
\end{aligned}
$$

Hence, if $\left(\alpha^{+}\right)$is satisfied, from (4.28) we immediately see that $H\left(\boldsymbol{Q}^{u}, \tau\right)>0$ if $\tau \leq \ln (\rho)$. Further, if $\boldsymbol{\Omega}^{-}$holds from (4.28) and (4.1), for any $\tau>\ln (R)$, we see that

$$
\begin{aligned}
H\left(\boldsymbol{Q}^{u}, \tau\right)= & \int_{-\infty}^{\ln (R)-\tau} \dot{\phi}(t+\tau) \frac{\left[x_{1}^{u}(t+\tau)\right]^{\sigma}}{\sigma} d t \\
& +\int_{\ln (R)-\tau}^{0} \dot{\phi}(t+\tau) \frac{\left[\underline{x}_{1}^{u}(t)\right]^{\sigma}}{\sigma} d t \\
\leq & \int_{-\infty}^{\ln (R)-\tau} \dot{\phi}^{+}(t+\tau) \frac{\left[\bar{x}_{1}^{u}(t)\right]^{\sigma}}{\sigma} d t \\
& -\int_{\ln (R)-\tau}^{0} \dot{\phi}^{-}(t+\tau) \frac{\left[\underline{x}_{1}^{u}(t)\right]^{\sigma}}{\sigma} d t .
\end{aligned}
$$


Then, using the asymptotic estimate in (4.1) and the steepness requirement on $K(r)$, we see that there is $\widetilde{T}>\mathrm{e}^{R}$ such that the right hand side of (4.29) is negative for any $\tau \geq \widetilde{T}$ (see [13, Lemma 3.1] for more details). The proof for $H\left(\boldsymbol{Q}^{s}, \tau\right)$ is analogous.

Now, we remove assumption (H1), however from the hypotheses of Lemma 4.2 (H2) follows, so we can still construct $\widehat{\boldsymbol{W}}^{\boldsymbol{u}}(\tau)$ and $\widehat{\boldsymbol{W}}^{\boldsymbol{s}}(\tau)$. Once again, if $\left(\alpha^{+}\right)$is satisfied from (4.28) we get $H\left(Q^{u}, \tau\right)>0$ if $\tau \leq \ln (\rho)$. When $\boldsymbol{\Omega}^{-}$holds we follow closely the argument of [13, §3]; so let $\overline{\boldsymbol{Q}}^{u}$ be a point in $\widehat{\boldsymbol{W}}^{\boldsymbol{u}}(\tau) \cap \overline{\boldsymbol{L}}^{+}$, see (4.9). Then we can control $\boldsymbol{x}\left(\tau, \overline{\boldsymbol{Q}}^{u} ; t\right)$ via $\underline{x}_{1}^{u}$ for $t \leq \tau$ (i.e. when $\boldsymbol{x}\left(\tau, \overline{\boldsymbol{Q}}^{u} ; t\right) \in \overline{\boldsymbol{E}}$ but not when $\boldsymbol{x}\left(\tau, \overline{\boldsymbol{Q}}^{u} ; t\right) \in \widetilde{\boldsymbol{E}}$ i.e. for $\left.t>\tau\right)$. Hence repeating the computation in (4.29) we find $\bar{T} \geq \mathrm{e}^{R}$ such that $H\left(\overline{\boldsymbol{Q}}^{u}, T\right)<0$ for any $T \geq \bar{T}$. Then, using the fact that $H\left(\boldsymbol{x}\left(T, \overline{\boldsymbol{Q}}^{\boldsymbol{u}} ; t\right), t\right)$ is decreasing in $t$ for $t \geq \mathrm{e}^{R}$ we find that $\boldsymbol{x}\left(T, \overline{\boldsymbol{Q}}^{u} ; t\right)$ intersects $\widehat{\boldsymbol{\ell}}$ at some $\tau>T$ in a point $\widetilde{\boldsymbol{Q}}^{\boldsymbol{u}}(\tau) \in \widetilde{\boldsymbol{\xi}}^{\boldsymbol{u}}(\tau)$ and $H\left(\widetilde{\boldsymbol{Q}}^{\boldsymbol{u}}(\tau), \tau\right)<0$. See [13, Lemmas 3.1, 3.2] for more details.

Now we proceed to prove Lemma 4.3, so we always assume that either (H1) or (H2) are satisfied so that we can construct $\widehat{\boldsymbol{W}}^{\boldsymbol{u}}(\tau)$ and $\widehat{\boldsymbol{W}}^{\boldsymbol{s}}(\tau)$ for any $\tau \in \mathbb{R}$, either via Lemma 3.2 or via Lemma 3.5. Further, we assume that (4.2) holds and, without loss of generality, $\tau_{1}<\tau_{2}$.

At page 221 in [12] we said that $\widehat{\boldsymbol{\zeta}}^{\boldsymbol{u}}:=\left\{(\boldsymbol{Q}, \tau) \mid \boldsymbol{Q} \in \widehat{\boldsymbol{\xi}}^{\boldsymbol{u}}(\tau)\right\}$ is connected: this result in general is false, and this is why Lemma 4.3 needs a non-trivial proof. In fact, assume first, for simplicity, (H1) and that (1.6) is smooth (e.g. $p=2$ ) so that $\widehat{\boldsymbol{W}}^{\boldsymbol{u}}(\tau) \subset \boldsymbol{E}^{+}$is a 1 dimensional manifold. Even in this case $\widehat{\boldsymbol{W}}^{\boldsymbol{u}}(\tau)$ may be tangent to $\widehat{\boldsymbol{\ell}}$ and $\widehat{\boldsymbol{\xi}}^{\boldsymbol{u}}(\tau)$ needs not be a singleton. Further, even if $\widehat{\boldsymbol{\xi}}^{\boldsymbol{u}}(\tau)=\left\{\boldsymbol{Q}^{\boldsymbol{u}}(\tau)\right\}$ is a singleton for any $\tau \in \mathbb{R}, \boldsymbol{Q}^{\boldsymbol{u}}(\tau)$ needs not be continuous in $\tau$ when $\widehat{\boldsymbol{W}}^{\boldsymbol{u}}(\tau)$ is tangent to $\widehat{\boldsymbol{\ell}}$.

However we can still prove Lemma 4.3. Assume that $\widehat{\boldsymbol{W}}^{\boldsymbol{u}}(\tau)$ and $\widehat{\boldsymbol{W}}^{\boldsymbol{s}}(\tau)$ are well defined (i.e. either (H1) or (H2) hold true), fix $\tau \in \mathbb{R}$ and for any $\boldsymbol{Q} \in \widehat{\boldsymbol{W}}^{u}(\tau), \boldsymbol{R} \in \widehat{\boldsymbol{W}}^{s}(\tau)$ set

$$
\begin{aligned}
\mathcal{T}_{\tau}^{u}(\boldsymbol{Q}) & =\sup \left\{T \mid \dot{x}_{1}(\tau, \boldsymbol{Q} ; t)>0 \text { when } t<T\right\}, \\
\mathcal{H}_{\tau}^{u}(\boldsymbol{Q}) & =H\left(\boldsymbol{x}\left(\tau, \boldsymbol{Q} ; \mathcal{T}_{\tau}^{u}(\boldsymbol{Q})\right), \mathcal{T}_{\tau}^{u}(\boldsymbol{Q})\right), \\
\mathcal{T}_{\tau}^{s}(\boldsymbol{R}) & =\inf \left\{T \mid \dot{x}_{1}(\tau, \boldsymbol{R} ; t)<0 \text { when } t>T\right\}, \\
\mathcal{H}_{\tau}^{s}(\boldsymbol{R}) & =H\left(\boldsymbol{x}\left(\tau, \boldsymbol{R} ; \mathcal{T}_{\tau}^{s}(\boldsymbol{R})\right), \mathcal{T}_{\tau}^{s}(\boldsymbol{R})\right) .
\end{aligned}
$$

Notice that

$$
\boldsymbol{x}(\tau, \boldsymbol{Q} ; t) \in \boldsymbol{E}^{+} \quad \text { for any } t<\mathcal{T}_{\tau}^{u}(\boldsymbol{Q}) \text { and that } \quad \boldsymbol{x}\left(\tau, \boldsymbol{Q} ; \mathcal{T}_{\tau}^{u}(\boldsymbol{Q})\right) \in \widehat{\boldsymbol{\ell}} .
$$

Analogously,

$$
\boldsymbol{x}(\tau, \boldsymbol{R} ; t) \in \boldsymbol{E}^{-} \quad \text { for any } t>\mathcal{T}_{\tau}^{s}(\boldsymbol{R}) \quad \text { and } \quad \boldsymbol{x}\left(\tau, \boldsymbol{R} ; \mathcal{T}_{\tau}^{u}(\boldsymbol{R})\right) \in \widehat{\boldsymbol{\ell}} .
$$

Then we have the following 
LEMmA 4.8. Assume (H1), then the functions

$$
\begin{aligned}
\boldsymbol{\Gamma}^{\boldsymbol{u}}: \widehat{\boldsymbol{W}}^{\boldsymbol{u}}\left(\tau_{1}\right) \rightarrow\left[\tau_{1} ;+\infty\right) \times \mathbb{R}, & \boldsymbol{\Gamma}^{\boldsymbol{u}}(\boldsymbol{Q}) & =\left(\mathcal{T}_{\tau_{1}}^{u}(\boldsymbol{Q}), \mathcal{H}_{\tau_{1}}^{u}(\boldsymbol{Q})\right), \\
\boldsymbol{\Gamma}^{\boldsymbol{s}}: \widehat{\boldsymbol{W}}^{\boldsymbol{s}}\left(\tau_{2}\right) \rightarrow\left(-\infty ; \tau_{2}\right] \times \mathbb{R}, & \boldsymbol{\Gamma}^{\boldsymbol{s}}(\boldsymbol{R}) & =\left(\mathcal{T}_{\tau_{2}}^{s}(\boldsymbol{R}), \mathcal{H}_{\tau_{2}}^{s}(\boldsymbol{R})\right),
\end{aligned}
$$

are continuous. Further the map $H(\cdot, \tau): \widehat{\ell} \rightarrow \mathbb{R}$, is injective for any $\tau \in \mathbb{R}$.

Proof. The continuity of $\mathcal{T}_{\tau_{1}}^{u}(\boldsymbol{Q})$ follows from the transversality of the flow of (1.6) on $\widehat{\ell}$; then the continuity of $\mathcal{H}_{\tau_{1}}^{u}(\cdot)$ immediately follows. The proof concerning $\mathcal{T}_{\tau_{2}}^{s}$ and $\mathcal{H}_{\tau}^{s}$ is analogous. The injectivity of $H(\cdot, \tau)$ follows from elementary computations: notice that $H(\cdot, \tau)$ has its minimum at $\boldsymbol{P}(\tau)$ and $\widehat{\boldsymbol{\ell}}$ lies on the right hand side of $\boldsymbol{P}(\tau)$.

Now we are ready to prove the following partial result.

Lemma 4.9. Assume (H1), then Lemma 4.3 holds.

Proof. Consider the stripe $\mathcal{S}:=\left\{(\tau, H) \mid \tau \geq \tau_{1}, H>H(\boldsymbol{P}(\tau), \tau)\right\}$. Assume first for simplicity that $\widehat{\boldsymbol{W}}^{\boldsymbol{u}}\left(\tau_{1}\right)$ and $\widehat{\boldsymbol{W}}^{\boldsymbol{s}}\left(\tau_{2}\right)$ are 1-dimensional manifolds (e.g. if $2 n /(n+2) \leq p \leq 2)$; then $\boldsymbol{B}^{\boldsymbol{u}}:=\left\{\boldsymbol{\Gamma}^{\boldsymbol{u}}(\boldsymbol{Q}) \mid \boldsymbol{Q} \in \widehat{\boldsymbol{W}}^{\boldsymbol{u}}\left(\tau_{1}\right)\right\}$ is a continuous path and splits $\mathcal{S}$ in two open subsets, one above $\boldsymbol{B}^{\boldsymbol{u}}$, say $\boldsymbol{A}^{\boldsymbol{u},+}$ and one below, say $\boldsymbol{A}^{\boldsymbol{u},-}$. Observe that the image of $\widehat{\boldsymbol{W}}^{\boldsymbol{s}}\left(\tau_{2}\right)$ through $\boldsymbol{\Gamma}^{\boldsymbol{s}}$ is a continuous path as well.

Assume to fix the ideas that $\tau_{1}<\tau_{2}$ in $(4.2)$; let $\boldsymbol{Q}^{\boldsymbol{u}}\left(\tau_{i}\right) \in \widehat{\boldsymbol{\xi}}^{\boldsymbol{u}}\left(\tau_{i}\right), \boldsymbol{Q}^{\boldsymbol{s}}\left(\tau_{i}\right) \in$ $\widehat{\boldsymbol{\xi}}^{\boldsymbol{s}}\left(\tau_{i}\right)$, for $i=1,2$. Observe that there are $\boldsymbol{R}^{\boldsymbol{u}} \in \widehat{\boldsymbol{W}}^{\boldsymbol{u}}\left(\tau_{1}\right)$ and $\boldsymbol{R}^{\boldsymbol{s}} \in \widehat{\boldsymbol{W}}^{\boldsymbol{s}}\left(\tau_{2}\right)$ such that $\boldsymbol{x}\left(\tau_{1}, \boldsymbol{R}^{\boldsymbol{u}} ; \tau_{2}\right)=\boldsymbol{Q}^{\boldsymbol{u}}\left(\tau_{2}\right)$, and $\boldsymbol{x}\left(\tau_{2}, \boldsymbol{R}^{\boldsymbol{s}} ; \tau_{1}\right)=\boldsymbol{Q}^{\boldsymbol{s}}\left(\tau_{1}\right)$. Hence, from (4.2), we find

$$
\boldsymbol{\Gamma}^{\boldsymbol{s}}\left(\boldsymbol{R}^{\boldsymbol{s}}\right)=\left(\tau_{1}, \mathcal{H}_{\tau_{2}}^{s}\left(\boldsymbol{R}^{\boldsymbol{s}}\right)\right), \quad \boldsymbol{\Gamma}^{\boldsymbol{u}}\left(\boldsymbol{Q}^{\boldsymbol{u}}\left(\tau_{1}\right)\right)=\left(\tau_{1}, \mathcal{H}_{\tau_{1}}^{u}\left(\boldsymbol{Q}^{\boldsymbol{u}}\left(\tau_{1}\right)\right)\right.
$$

where

$$
\mathcal{H}_{\tau_{2}}^{s}\left(\boldsymbol{R}^{\boldsymbol{s}}\right)=H\left(\boldsymbol{Q}^{\boldsymbol{s}}\left(\tau_{1}\right), \tau_{1}\right)<0<H\left(\boldsymbol{Q}^{\boldsymbol{u}}\left(\tau_{1}\right), \tau_{1}\right)=\mathcal{H}_{\tau_{1}}^{u}\left(\boldsymbol{Q}^{\boldsymbol{u}}\left(\tau_{1}\right)\right) .
$$

Therefore $\boldsymbol{\Gamma}^{\boldsymbol{s}}\left(\boldsymbol{R}^{\boldsymbol{s}}\right) \in \boldsymbol{A}^{\boldsymbol{u},-}$. Analogously, we find

$$
\boldsymbol{\Gamma}^{\boldsymbol{s}}\left(\boldsymbol{Q}^{\boldsymbol{s}}\left(\tau_{2}\right)\right)=\left(\tau_{2}, \mathcal{H}_{\tau_{2}}^{s}\left(\boldsymbol{Q}^{\boldsymbol{s}}\left(\tau_{2}\right)\right), \quad \boldsymbol{\Gamma}^{\boldsymbol{u}}\left(\boldsymbol{R}^{\boldsymbol{u}}\right)=\left(\tau_{2}, \mathcal{H}_{\tau_{1}}^{u}\left(\boldsymbol{R}^{\boldsymbol{u}}\right)\right)\right.
$$

where

$$
\mathcal{H}_{\tau_{1}}^{u}\left(\boldsymbol{R}^{\boldsymbol{u}}\right)=H\left(\boldsymbol{Q}^{\boldsymbol{u}}\left(\tau_{2}\right), \tau_{2}\right)<0<H\left(\boldsymbol{Q}^{\boldsymbol{s}}\left(\tau_{2}\right), \tau_{2}\right)=\mathcal{H}_{\tau_{2}}^{s}\left(\boldsymbol{Q}^{\boldsymbol{s}}\left(\tau_{2}\right)\right) .
$$

Therefore $\boldsymbol{\Gamma}^{\boldsymbol{s}}\left(\boldsymbol{Q}^{\boldsymbol{s}}\left(\tau_{2}\right)\right) \in \boldsymbol{A}^{\boldsymbol{u},+}$.

Hence, from a continuity argument, we see that there is $\boldsymbol{R}^{*} \in \widehat{\boldsymbol{W}}^{\boldsymbol{s}}\left(\tau_{2}\right)$ such that $\boldsymbol{\Gamma}^{\boldsymbol{s}}\left(\boldsymbol{R}^{*}\right) \in \boldsymbol{B}^{\boldsymbol{u}}$. Then, by construction, there is $\tau^{*} \in\left(\tau_{1}, \tau_{2}\right)$ such that

$$
\boldsymbol{x}\left(\tau_{2}, \boldsymbol{R}^{*} ; \tau^{*}\right)=\boldsymbol{Q}^{*} \in \widetilde{\boldsymbol{\xi}}^{\boldsymbol{u}}\left(\tau^{*}\right) \cap \widetilde{\boldsymbol{\xi}}^{\boldsymbol{s}}\left(\tau^{*}\right) .
$$


Hence, from Lemma 4.1, we see that $\boldsymbol{x}\left(\tau_{2}, \boldsymbol{R}^{*} ; t\right) \in \boldsymbol{E}^{+}\left(\tau^{*}\right)$ for any $t \leq \tau^{*}$ and $\boldsymbol{x}\left(\tau_{2}, \boldsymbol{R}^{*} ; t\right) \in \boldsymbol{E}^{-}\left(\tau^{*}\right)$ for any $t \geq \tau^{*}$, and the corresponding solution $u(r)$ of (1.3) is a GS with FD.

Now we consider the general case, where $\widehat{\boldsymbol{W}}^{\boldsymbol{u}}\left(\tau_{1}\right)$ and $\widehat{\boldsymbol{W}}^{s}\left(\tau_{2}\right)$ are just continua, cf. Theorem 3.2. In this case $\boldsymbol{B}^{\boldsymbol{u}}$ is a continuum as well so, for any $\varepsilon>0$, there is a path, say $\boldsymbol{\gamma}^{\varepsilon}$, having $\boldsymbol{\Gamma}^{\boldsymbol{u}}(0,0)$ and $\boldsymbol{\Gamma}^{\boldsymbol{u}}\left(\boldsymbol{Q}^{\boldsymbol{u}}\left(\tau_{1}\right)\right)$ as endpoints and such that

$$
\boldsymbol{\gamma}^{\varepsilon} \subset B\left(\boldsymbol{B}^{u}, \varepsilon\right):=\left\{\boldsymbol{Q} \mid\|\boldsymbol{Q}-\boldsymbol{R}\| \leq \varepsilon, \boldsymbol{R} \in \boldsymbol{B}^{\boldsymbol{u}}\right\} .
$$

Hence $B\left(\boldsymbol{B}^{\boldsymbol{u}}, \varepsilon\right)$ splits $\mathcal{S}$ in two open subsets $\boldsymbol{A}^{\boldsymbol{u},+}$ and $\boldsymbol{A}^{\boldsymbol{u},-}$. We emphasize that using (4.2) we can reprove (4.31) and (4.32), and we conclude by repeating the previous argument with trivial adaptations.

Lemma 4.10. Assume (H2), then the function $\boldsymbol{\Gamma}^{\boldsymbol{u}}(\boldsymbol{Q})$ and $\boldsymbol{\Gamma}^{\boldsymbol{s}}(\boldsymbol{R})$ are continuous as long as $\boldsymbol{\Gamma}^{u}(\boldsymbol{Q}) \in \mathcal{S}\left(\right.$ i.e. $\left.\mathcal{H}_{\tau_{1}}^{u}(\boldsymbol{Q})>H\left(\boldsymbol{P}\left(\mathcal{T}_{\tau_{1}}^{u}(\boldsymbol{Q})\right), \mathcal{T}_{\tau_{1}}^{u}(\boldsymbol{Q})\right)\right)$ and $\boldsymbol{\Gamma}^{\boldsymbol{s}}(\boldsymbol{R}) \in \mathcal{S}$ (i.e. $\mathcal{H}_{\tau_{2}}^{s}(\boldsymbol{R})>H\left(\boldsymbol{P}\left(\mathcal{T}_{\tau_{2}}^{s}(\boldsymbol{R}), \mathcal{T}_{\tau_{2}}^{s}(\boldsymbol{R})\right)\right.$, respectively.

Proof. We just consider the case of $\boldsymbol{Q} \in \widehat{\boldsymbol{W}}^{\boldsymbol{u}}\left(\tau_{1}\right)$, since the case of $\boldsymbol{R} \in$ $\widehat{\boldsymbol{W}}^{\boldsymbol{s}}\left(\tau_{2}\right)$ is analogous. Observe that the trajectory $\boldsymbol{x}\left(\tau_{1}, \boldsymbol{Q} ; t\right)$ either crosses $\widehat{\boldsymbol{\ell}}$ transversally at $t=\mathcal{T}_{\tau_{1}}^{u}(\boldsymbol{Q})$ on the right of $\boldsymbol{P}\left(\mathcal{T}_{\tau_{1}}^{u}(\boldsymbol{Q})\right)$ or it reaches $\boldsymbol{P}\left(\mathcal{T}_{\tau_{1}}^{u}(\boldsymbol{Q})\right)$ at $t=\mathcal{T}_{\tau_{1}}^{u}(\boldsymbol{Q})$. In the former case the function $\mathcal{T}_{\tau_{1}}^{u}(\boldsymbol{Q})$ is continuous (thanks to the transversality of the crossing), hence $\Gamma^{\boldsymbol{u}}(\boldsymbol{Q})$ is continuous too and

$$
\mathcal{H}_{\tau_{1}}^{u}(\boldsymbol{Q})>H\left(\boldsymbol{P}\left(\mathcal{T}_{\tau_{1}}^{u}(\boldsymbol{Q})\right), \mathcal{T}_{\tau_{1}}^{u}(\boldsymbol{Q})\right)
$$

and the lemma is proved. By the way we observe that in the latter case $\boldsymbol{\Gamma}^{\boldsymbol{u}}(\boldsymbol{Q})$ may be discontinuous but $\mathcal{H}_{\tau_{1}}^{u}(\boldsymbol{Q})=H\left(\boldsymbol{P}\left(\mathcal{T}_{\tau_{1}}^{u}(\boldsymbol{Q})\right), \mathcal{T}_{\tau_{1}}^{u}(\boldsymbol{Q})\right)$.

Proof of Lemma 4.3. If (H1) holds Lemma 4.3 follows from Lemma 4.9, so we assume (H2). Assume again first that $\widehat{\boldsymbol{W}}^{u}\left(\tau_{1}\right)$ and $\widehat{\boldsymbol{W}}^{\boldsymbol{s}}\left(\tau_{2}\right)$ are 1-dimensional manifold, e.g. when $2 n /(n+2) \leq p \leq 2$.

We distinguish two cases. Firstly, if the image $\boldsymbol{B}^{\boldsymbol{u}}$ of $\boldsymbol{\Gamma}^{\boldsymbol{u}}$ is contained in $\mathcal{S}$ it follows that $\boldsymbol{\Gamma}^{\boldsymbol{u}}$ is continuous, hence $\boldsymbol{B}^{\boldsymbol{u}}$ is a continuous path and splits $\mathcal{S}$ in two open subsets $\boldsymbol{A}^{\boldsymbol{u},+}$ and $\boldsymbol{A}^{\boldsymbol{u},-}$, respectively above and below $\boldsymbol{B}^{\boldsymbol{u}}$. Secondly, if there is $\overline{\boldsymbol{Q}} \in \widehat{\boldsymbol{W}}^{\boldsymbol{u}}\left(\tau_{1}\right)$ such that $\boldsymbol{\Gamma}^{\boldsymbol{u}}(\overline{\boldsymbol{Q}}) \notin \mathcal{S}$, then $\boldsymbol{\Gamma}^{\boldsymbol{u}}(\overline{\boldsymbol{Q}})$ lies on the curve $\tau \rightarrow$ $(\tau, H(\boldsymbol{P}(\tau), \tau))$. We denote by $\boldsymbol{w}^{\boldsymbol{u}}$ the connected component of $\boldsymbol{\Gamma}^{\boldsymbol{u}}\left(\widehat{\boldsymbol{W}}^{\boldsymbol{u}}\left(\tau_{1}\right)\right)$ (a subpath if $\widehat{\boldsymbol{W}}^{\boldsymbol{u}}\left(\tau_{1}\right)$ is a manifold) which contains $\boldsymbol{\Gamma}^{\boldsymbol{u}}\left(\boldsymbol{Q}^{\boldsymbol{u}}\left(\tau_{1}\right)\right)$ and has $\boldsymbol{\Gamma}^{\boldsymbol{u}}(\overline{\boldsymbol{Q}})$ in its border. By construction $\boldsymbol{w}^{\boldsymbol{u}}$ splits again $\mathcal{S}$ in two open subsets, say $\boldsymbol{A}^{\boldsymbol{u},+}$ and $\boldsymbol{A}^{\boldsymbol{u},-}$, above and below $\boldsymbol{B}^{\boldsymbol{u}}$.

As in the proof of Lemma 4.9 let $\boldsymbol{Q}^{\boldsymbol{u}}\left(\tau_{2}\right) \in \widehat{\boldsymbol{\xi}}^{\boldsymbol{u}}\left(\tau_{2}\right), \boldsymbol{Q}^{\boldsymbol{s}}\left(\tau_{1}\right) \in \widehat{\boldsymbol{\xi}}^{\boldsymbol{s}}\left(\tau_{1}\right)$, and let $\boldsymbol{R}^{\boldsymbol{u}} \in \widehat{\boldsymbol{W}}^{\boldsymbol{u}}\left(\tau_{1}\right)$ and $\boldsymbol{R}^{\boldsymbol{s}} \in \widehat{\boldsymbol{W}}^{\boldsymbol{s}}\left(\tau_{2}\right)$ be the points such that $\boldsymbol{x}\left(\tau_{1}, \boldsymbol{R}^{\boldsymbol{u}} ; \tau_{2}\right)=$ $\boldsymbol{Q}^{\boldsymbol{u}}\left(\tau_{2}\right)$, and $\boldsymbol{x}\left(\tau_{2}, \boldsymbol{R}^{\boldsymbol{s}} ; \tau_{1}\right)=\boldsymbol{Q}^{\boldsymbol{s}}\left(\tau_{1}\right)$. Using again (4.2) we find $\mathcal{H}_{\tau_{2}}^{s}\left(\boldsymbol{R}^{\boldsymbol{s}}\right)<0<$ $\mathcal{H}_{\tau_{1}}^{u}\left(\boldsymbol{Q}^{\boldsymbol{u}}\left(\tau_{1}\right)\right)$, hence

$$
\boldsymbol{\Gamma}^{\boldsymbol{s}}\left(\boldsymbol{R}^{\boldsymbol{s}}\right)=\left(\tau_{1}, \mathcal{H}_{\tau_{2}}^{s}\left(\boldsymbol{R}^{\boldsymbol{s}}\right)\right)=\left(\tau_{1}, H\left(\boldsymbol{Q}^{\boldsymbol{s}}\left(\tau_{1}\right), \tau_{1}\right)\right) \in \boldsymbol{A}^{\boldsymbol{u},-} .
$$


Further, from (4.2) we also find that

$$
\boldsymbol{\Gamma}^{\boldsymbol{s}}\left(\boldsymbol{Q}^{\boldsymbol{s}}\left(\tau_{2}\right)\right)=\left(\tau_{2}, H\left(\boldsymbol{Q}^{\boldsymbol{s}}\left(\tau_{2}\right), \tau_{2}\right) \in \boldsymbol{A}^{\boldsymbol{u},+} .\right.
$$

Let us denote by $\boldsymbol{B}^{\boldsymbol{s}}:=\boldsymbol{\Gamma}^{\boldsymbol{s}}\left(\widehat{\boldsymbol{W}}^{\boldsymbol{s}}\left(\tau_{2}\right)\right)$ : we claim that there is $\boldsymbol{R}^{*} \in \widehat{\boldsymbol{W}}^{\boldsymbol{s}}\left(\tau_{2}\right)$ such that $\boldsymbol{\Gamma}^{\boldsymbol{s}}\left(\boldsymbol{R}^{*}\right) \in\left(\boldsymbol{B}^{\boldsymbol{u}} \cap \boldsymbol{B}^{\boldsymbol{s}}\right)$. Then, from Lemma 4.1, we see that the corresponding solution $u(r)$ of $(1.3)$ is a GS with FD.

Now we prove the claim. Assume first that $\boldsymbol{B}^{s} \subset \mathcal{S}$, so that $\boldsymbol{\Gamma}^{s}$ is continuous. Then, from a continuity argument, we see that there is $\boldsymbol{R}^{*} \in \widehat{\boldsymbol{W}}^{\boldsymbol{s}}\left(\tau_{2}\right)$ such that $\Gamma^{s}\left(\boldsymbol{R}^{*}\right) \in\left(\boldsymbol{B}^{u} \cap \boldsymbol{B}^{s}\right)$.

Assume now that $\boldsymbol{B}^{\boldsymbol{s}} \not \subset \mathcal{S}$. From Lemma 4.10 we see that there is $\overline{\boldsymbol{R}}^{\boldsymbol{s}} \in$ $\widehat{\boldsymbol{W}}^{\boldsymbol{s}}\left(\tau_{2}\right)$ such that $\mathcal{T}_{\tau_{2}}^{s}\left(\overline{\boldsymbol{R}}^{\boldsymbol{s}}\right)=\bar{T}$ and $\boldsymbol{x}\left(\tau_{2}, \overline{\boldsymbol{R}}^{\boldsymbol{s}} ; \bar{T}\right)=\boldsymbol{P}(\bar{T})$. Denote by $\boldsymbol{w}^{\boldsymbol{s}}$ the connected component of $\boldsymbol{B}^{\boldsymbol{s}}$ (a subpath if $\widehat{\boldsymbol{W}}^{\boldsymbol{s}}\left(\tau_{2}\right)$ is a manifold) which contains $\boldsymbol{\Gamma}^{\boldsymbol{s}}\left(\boldsymbol{Q}^{\boldsymbol{s}}\left(\tau_{2}\right)\right)$ and has $\boldsymbol{\Gamma}^{\boldsymbol{s}}\left(\overline{\boldsymbol{R}}^{\boldsymbol{s}}\right)$ in its border. Notice that by construction $\boldsymbol{\Gamma}^{\boldsymbol{s}}\left(\overline{\boldsymbol{R}}^{\boldsymbol{s}}\right)=$ $(\bar{T}, H(\boldsymbol{P}(\bar{T}), \bar{T}))$ either belongs to $\boldsymbol{w}^{\boldsymbol{u}}$ or it belongs to $\boldsymbol{A}^{\boldsymbol{u},-}$. In the former case we have found an intersection between $\boldsymbol{w}^{\boldsymbol{u}}$ and $\boldsymbol{w}^{\boldsymbol{s}}$ so the claim is proved; in the latter case from a continuity argument we get an intersection between $\boldsymbol{w}^{\boldsymbol{u}}$ and $\boldsymbol{w}^{\boldsymbol{s}}$, since $\boldsymbol{\Gamma}^{\boldsymbol{s}}\left(\boldsymbol{Q}^{\boldsymbol{s}}\left(\tau_{2}\right)\right) \in \boldsymbol{A}^{\boldsymbol{u},+}$, and the claim is proved.

Now we consider the general case where $\widehat{\boldsymbol{W}}^{\boldsymbol{u}}\left(\tau_{1}\right)$ and $\widehat{\boldsymbol{W}}^{\boldsymbol{s}}\left(\tau_{2}\right)$ are just continua. Reasoning as at the end of the proof of Lemma 4.9 we define the path $\gamma^{\varepsilon}$ having $\boldsymbol{\Gamma}^{\boldsymbol{u}}((0,0))$ and $\boldsymbol{\Gamma}^{\boldsymbol{u}}\left(\boldsymbol{Q}^{\boldsymbol{u}}\left(\tau_{1}\right)\right)$ as endpoints and $B\left(\boldsymbol{B}^{\boldsymbol{u}}, \varepsilon\right)$ as in (4.33). Then we denote by $\boldsymbol{A}^{\boldsymbol{u},+}$ and $\boldsymbol{A}^{\boldsymbol{u},-}$ the two open subsets in which $B\left(\boldsymbol{B}^{\boldsymbol{u}}, \varepsilon\right)$ splits $\mathcal{S}$. Then we conclude by repeating the previous argument with trivial changes. This concludes the proof of the lemma.

\section{REFERENCES}

[1] F. Battelli And R. Johnson, On positive solutions of the scalar curvature equation when the curvature has variable sign, Nonlinear Anal. 47 (2001), 1029-1037.

[2] G. Bianchi AND H. Egnell, $A$ variational approach to the equation $\Delta u+$ $K u^{(n+2) /(n-2)}=0$ in $\mathbb{R}^{n}$, Arch. Ration. Mech. Anal. 122 (1993), 159-182.

[3] G. BiAnchi, Non-existence and symmetry of solutions to the scalar curvature equation, Comm. Partial Differential Equations 21 (1996), 229-234.

[4] M.F. Bidaut-VÉron, Local and global behavior of solutions of quasilinear equations of Emden-Fowler type, Arch. Ration. Mech. Anal. 107 (1989), 293-324.

[5] L. Caffarelli, B. Gidas and J. Spruck, Asymptotic symmetry and local behavior of semilinear elliptic equations with critical sobolev growth, Comm. Pur. Appl. Math. 42 (1989), no. 3, 271-297.

[6] C.C. Chen And C.S. Lin, Blowing up with infinite energy of conformal metrics on $S^{n}$, Comm. Partial Differential Equations 24 (1999), 785-799.

[7] C.C. Chen AND C.S. Lin, On the asymptotic symmetry of singular solutions of the scalar curvature equations, Math. Ann. 313 (1999), 229-245.

[8] E. Coddington and N. Levinson, Theory of Ordinary Differential Equations, Mc Graw Hill, New York, 1955.

[9] F. Dalbono And M. Franca, Nodal solutions for supercritical Laplace equations, Comm. Math. Phys. 347 (2016), 875-901. 
[10] W.Y. Ding AND W.M. NI, On the elliptic equation $\Delta u+K u^{(n+2) /(n-2)}=0$ and related topics, Duke Math. J. 52 (1985), 485-506.

[11] I. Flores and M. Franca, Multiplicity results for the scalar curvature equation, J. Differential Equations 259 (2015), 4327-4355.

[12] M. FrancA, Non-autonomous quasilinear elliptic equations and Ważewski's principle, Topol. Methods Nonlinear Anal. 23 (2004), 213-238.

[13] M. Franca, Structure theorems for positive radial solutions of the generalized scalar curvature equation, Funkc. Ekv. 52 (2009), 343-369.

[14] M. Franca And R. Johnson, Ground states and singular ground states for quasilinear partial differential equations with critical exponent in the perturbative case, Adv. Nonlinear Stud. 4 (2004), 93-120.

[15] B. Gidas, W.M. Ni And L. Nirenberg, Symmetry and related properties via the maximum principle, Comm. Math. Phys. 68 (1979), no. 3, 209-243.

[16] R. Johnson, X.B. PAN AND Y.F. YI, The Melnikov method and elliptic equations with critical exponent, Indiana Math. J. 43 (1994), 1045-1077.

[17] R. Johnson, X.B. PAn And Y.F. YI, Singular ground states of semilinear elliptic equations via invariant manifold theory, Nonlinear Anal. 20 (1993), 1279-1302.

[18] N. Kawano, W.M. Ni and S. Yotsutani, A generalized Pohozaev identity and its applications, J. Math. Soc. Japan 42 (1990), 541-564.

[19] N. Kawano, E. Yanagida and S. Yotsutani, Structure theorems for positive radial solutions to $\operatorname{div}\left(|D u|^{m-2} D u\right)+K(|x|) u^{q}=0$ in $\mathbb{R}^{n}$, J. Math. Soc. Japan 45 (1993), 719-742.

[20] D. Papini And F. ZAnolin, Periodic points and chaotic-like dynamics of planar maps associated to nonlinear Hill's equations with indefinite weight, Georgian Math. J. 9 (2002), 339-366.

[21] P. PUCCI AND R. SERVADEI, Existence, non-existence and regularity of radial ground states for -Laplacain equations with singular weights, Ann. Inst. H. Poincaré Anal. Non Linéaire 25 (2008), 505-537.

[22] B. ScIunzI, Classification of positive $D^{1, p}\left(\mathbb{R}^{n}\right)$-solutions to the critical p-Laplace equation in $\mathbb{R}^{n}$, Adv. Math. 291 (2016), 12-23.

[23] J. WeI AND S. YAN, Infinitely many solutions for the prescribed scalar curvature problem on $\mathbb{S}^{N}$, J. Funct. Anal. 258 (2010), 3048-3081.

[24] S. YAN, Concentration of solutions for the scalar curvature equation on $\mathbb{R}^{N}$, J. Differential Equations 163 (2000), 239-264.

[25] E. Yanagida And S. Yotsutani, Global structure of positive solutions to equations of Matukuma type, Arch. Rational Mech. Anal. 134 (1996), 199-226.

Manuscript received MONTH 00, 0000 accepted MONTH 00, 0000

\author{
Matteo Franca \\ Dipartimento di Ingegneria Industriale e Scienze Matematiche \\ Università Politecnica delle Marche \\ Via Brecce Bianche 1 \\ 60131 Ancona, ITALY \\ E-mail address: franca@dipmat.univpm.it
}

TMNA : VOLUME $00-0000-\mathrm{N}^{\mathrm{O}} 00$ 